TRANSACTIONS OF THE

AMERICAN MATHEMATICAL SOCIETY

Volume 348, Number 4, April 1996

\title{
FURTHER NICE EQUATIONS FOR NICE GROUPS
}

\author{
SHREERAM S. ABHYANKAR
}

\begin{abstract}
Nice sextinomial equations are given for unramified coverings of the affine line in nonzero characteristic $p$ with $\mathrm{P} \Omega^{-}(2 m, q)$ and $\Omega^{-}(2 m, q)$ as Galois groups where $m>3$ is any integer and $q>1$ is any power of $p>2$.
\end{abstract}

\section{INTRODUCTION}

Let $m>3$ be any integer, let $q>1$ be any power of a prime $p>2$, consider the polynomials $F^{-}=F^{-}(Y)=Y^{n}+T^{q^{2}} Y^{u^{\prime}}+X^{q} Y^{u}-X Y^{w}-T Y^{w^{\prime}}-1$ and $F^{*}=F^{*}(Y)=Y^{n^{*}}+X Y+1$ in indeterminates $T, X, Y$ over an algebraically closed field $k$ of characteristic $p$, where $n=1+q+\cdots+q^{2 m-1}, u^{\prime}=1+q+\cdots+q^{m+1}, u=$ $1+q+\cdots+q^{m}, w=1+q+\cdots+q^{m-2}, w^{\prime}=1+q+\cdots+q^{m-3}, n^{*}=1+q+\cdots+q^{m-1}$, and consider their respective Galois groups $\operatorname{Gal}\left(F^{-}, k(X, T)\right)$ and $\operatorname{Gal}\left(F^{*}, k(X)\right)$. Both these are special cases of the families of polynomials giving unramified coverings of the affine line in nonzero characteristic which were written down in my 1957 paper [A01]. In my "Nice Equations" paper [A04], as a consequence of Cameron-Kantor Theorem I [CaK] on antiflag transitive collineation groups, I proved that $\operatorname{Gal}\left(F^{*}, k(X)\right)=$ the projective special linear group $\operatorname{PSL}(m, q)$. In the present paper, as a consequence of Kantor's characterization of Rank 3 groups in terms of their subdegrees [Kan], supplemented by Cameron-Kantor Theorem IV $[\mathrm{CaK}]$, I shall show that $\operatorname{Gal}\left(F^{-}, k(X, T)\right)=$ the projective negative orthogonal group $\mathrm{P} \Omega^{-}(2 m, q) .{ }^{1}$ Note that Kantor's Rank 3 characterization depends on the Buekenhout-Shult characterization of polar spaces [BuS] which itself depends on Tits' classification of spherical buildings [Tit]. Recall that the Rank of a transitive permutation group is the number of orbits of its 1-point stabilizer, and the sizes of these orbits are called subdegrees.

As a corollary of the above theorem that the Galois group of $F^{-}$is $\mathrm{P} \Omega^{-}(2 m, q), \mathrm{I}$ shall show that the Galois group of a more general polynomial $f^{-}$is also $\mathrm{P} \Omega^{-}(2 m, q)$. Moreover, by slightly changing $f^{-}$and $F^{-}$, I shall show that we get polynomials $\phi^{-}$and $\phi_{2}^{-}$whose Galois group is the negative orthogonal group $\Omega^{-}(2 m, q)$. The polynomials $f^{-}, \phi^{-}$and $\phi_{2}^{-}$are also special cases of the families of polynomials giving unramified coverings of the affine line in nonzero characteristic written down in $[\mathrm{A} 01]$.

Received by the editors March 23, 1995.

1991 Mathematics Subject Classification. Primary 12F10, 14H30, 20D06, 20E22.

This work was partly supported by NSF grant DMS 91-01424 and NSA grant MDA 904-92$\mathrm{H}-3035$.

${ }^{1}$ The projective negative (resp: positive) orthogonal group $\mathrm{P} \Omega^{-}(2 m, q)$ (resp: $\left.\mathrm{P} \Omega^{+}(2 m, q)\right)$ is also called the projective elliptic (resp: hyperbolic) orthogonal group. 
As in [A03] and [A04], here the basic techniques will be MTR (the Method of Throwing away Roots) and FTP (Factorization of Polynomials).

It is a pleasure to thank Bill Kantor and Ulrich Meierfrankenfeld for inspiring conversations about this paper.

\section{Notation and Outline}

Let $k_{p}$ be a field of characteristic $p>0$, let $q>1$ be any power of $p$, and let $m>1$ be any integer. ${ }^{2}$ To abbreviate frequently occurring expressions, for every integer $i \geq-1$ we put

$$
\left.\langle i\rangle=1+q+q^{2}+\cdots+q^{i} \text { (convention: }\langle 0\rangle=1 \text { and }\langle-1\rangle=0\right) .
$$

We shall frequently use the geometric series identity

$$
1+Z+Z^{2}+\cdots+Z^{i}=\frac{Z^{i+1}-1}{Z-1}
$$

and its corollary

$$
\langle i\rangle=1+q+q^{2}+\cdots+q^{i}=\frac{q^{i+1}-1}{q-1} .
$$

Let

$$
f^{-}=f^{-}(Y)=Y^{\langle 2 m-1\rangle}-1+\sum_{i=1}^{m-1}\left(T_{i}^{q^{i}} Y^{\langle m-1+i\rangle}-T_{i} Y^{\langle m-1-i\rangle}\right)
$$

and note that then $f^{-}$is a monic polynomial of degree $\langle 2 m-1\rangle=1+q+q^{2}+$ $\cdots+q^{2 m-1}$ in $Y$ with coefficients in the polynomial $\operatorname{ring} k_{p}\left[T_{1}, \ldots, T_{m-1}\right]$. Now the constant term of $f^{-}$is -1 and the $Y$-exponent of every other term in $f^{-}$ is 1 modulo $p$, and hence $f^{-}-Y f_{Y}^{-}=-1$ where $f_{Y}^{-}$is the $Y$-derivative of $f^{-}$. Therefore $\operatorname{Disc}_{Y}\left(f^{-}\right)=-1$ where $\operatorname{Disc}_{Y}\left(f^{-}\right)$is the $Y$-discriminant of $f^{-}$, and hence the Galois group $\operatorname{Gal}\left(f^{-}, k_{p}\left(T_{1}, \ldots, T_{m-1}\right)\right)$ is well-defined as a subgroup of the symmetric group $\operatorname{Sym}_{\langle 2 m-1\rangle}$.

For $1 \leq e \leq m-1$, let $f_{e}^{-}$be obtained by substituting $T_{i}=0$ for all $i>e$ in $f^{-}$, i.e., let

$$
f_{e}^{-}=f_{e}^{-}(Y)=Y^{\langle 2 m-1\rangle}-1+\sum_{i=1}^{e}\left(T_{i}^{q^{i}} Y^{\langle m-1+i\rangle}-T_{i} Y^{\langle m-1-i\rangle}\right)
$$

and note that then $f_{e}^{-}$is a monic polynomial of degree $\langle 2 m-1\rangle=1+q+q^{2}+\cdots+$ $q^{2 m-1}$ in $Y$ with coefficients in the polynomial ring $k_{p}\left[T_{1}, \ldots, T_{e}\right]$ and, as above, $\operatorname{Disc}_{Y}\left(f_{e}^{-}\right)=-1$ and the Galois group $\operatorname{Gal}\left(f_{e}^{-}, k_{p}\left(T_{1}, \ldots, T_{e}\right)\right)$ is a subgroup of $\operatorname{Sym}_{\langle 2 m-1\rangle}$. Note that if $m>2$ and $k=k_{p}=$ an algebraically closed field (of characteristic $p>0$ ), then $F^{-}$is obtained by substituting $X, T$ for $T_{1}, T_{2}$ in $f_{2}^{-}$ and hence $\operatorname{Gal}\left(F^{-}, k(X, T)\right)=\operatorname{Gal}\left(f_{2}^{-}, k_{p}\left(T_{1}, T_{2}\right)\right)$.

\footnotetext{
${ }^{2}$ In the Abstract and the Introduction we assumed $p>2$ and $m>3$. But in the rest of the paper, unless stated otherwise, we only assume $p>0$ and $m>1$.
} 
In Section 3, we factor $f^{-}$as $f^{-}=\bar{f} f^{*}$ where $\bar{f}=\bar{f}(Y)$ and $f^{*}=f^{*}(Y)$ are monic polynomials of degrees $\left(q^{m}+1\right)\langle m-2\rangle$ and $q^{m-1}\left(q^{m}+1\right)$ in $Y$ with coefficients in $k_{p}\left[T_{1}, \ldots, T_{m-1}\right]$, respectively, and in case of $p \neq 2$ we factor $f^{*}$ further as $f^{*}=f^{* *} f^{* * *}$ where $f^{* *}=f^{* *}(Y)$ and $f^{* * *}=f^{* * *}(Y)$ are both monic polynomials of degree $q^{m-1}\left(q^{m}+1\right) / 2$ in $Y$ with coefficients in $k_{p}\left[T_{1}, \ldots, T_{m-1}\right]$. In Section 3 , we show that if $p=2$ then $\bar{f}$ and $f^{*}$ are irreducible in $k_{p}\left(T_{1}, \ldots, T_{m-1}\right)[Y]$, and if $p \neq 2$ then $\bar{f}, f^{* *}$ and $f^{* * *}$ are irreducible in $k_{p}\left(T_{1}, \ldots, T_{m-1}\right)[Y]$. Given any $e$ with $1 \leq e \leq m-1$, by putting $T_{i}=0$ for all $i>e$ in $\bar{f}$ and $f^{*}$ we get $f_{e}^{-}=\bar{f}_{e} f_{e}^{*}$ where $\overline{\bar{f}}_{e}$ and $f_{e}^{*}$ are monic polynomials of degrees $\left(q^{m}+1\right)\langle m-2\rangle$ and $q^{m-1}\left(q^{m}+1\right)$ in $Y$ with coefficients in $k_{p}\left[T_{1}, \ldots, T_{e}\right]$ respectively. Likewise, if $p \neq 2$ then by putting $T_{i}=0$ for all $i>e$ in $f^{* *}$ and $f^{* * *}$ we get $f_{e}^{*}=f_{e}^{* *} f_{e}^{* * *}$ where $f_{e}^{* *}$ and $f_{e}^{* * *}$ are both monic polynomials of degree $q^{m-1}\left(q^{m}+1\right) / 2$ in $Y$ with coefficients in $k_{p}\left[T_{1}, \ldots, T_{m-1}\right]$. In Section 3 , we also show that if $p=2$ then $\bar{f}_{e}$ and $f_{e}^{*}$ are irreducible in $k_{p}\left(T_{1}, \ldots, T_{e}\right)[Y]$, and if $p \neq 2$ then $\bar{f}_{e}, f_{e}^{* *}$ and $f_{e}^{* * *}$ are irreducible in $k_{p}\left(T_{1}, \ldots, T_{e}\right)[Y]$.

In Section 4 , we throw away a root of $\bar{f}$ to get its twisted derivative $f^{\prime}(Y, Z)$, and we let $g(Y, Z)$ be the polynomial obtained by first dividing the $Z$-roots of $f^{\prime}(Y, Z)$ by $Y$ and then changing $Y$ to $1 / Y$. Assuming $m>2$, in Section 4 , we factor $g(Y, Z)$ into two factors; to motivate the calculations, we first do this for $m=3$. The $Z$-degrees of these factors turn out to be $q\left(q^{m-1}+1\right)\langle m-3\rangle$ and $q^{2 m-2}$. In Section 4 , assuming $m>2$, we show that these factors are irreducible in case of $\bar{f}_{2}$ and hence also in case of $\bar{f}$ and $\bar{f}_{e}$ for $2 \leq e \leq m-1$, and therefore $\operatorname{Gal}\left(\bar{f}, k_{p}\left(T_{1}, \ldots, T_{m-1}\right)\right)$ and $\operatorname{Gal}\left(\bar{f}_{e}, k_{p}\left(T_{1}, \ldots, T_{e}\right)\right)$ for $2 \leq e \leq m-1$ are Rank 3 groups with subdegrees $1, q\left(q^{m-1}+1\right)\langle m-3\rangle$ and $q^{2 m-2}$. In Section 6 , from this Rank 3 description, we deduce the result that if $m>3 \leq p$ and $k_{p}$ is algebraically closed then $\operatorname{Gal}\left(f^{-}, k_{p}\left(T_{1}, \ldots, T_{m-1}\right)\right)=\operatorname{Gal}\left(f_{e}^{-}, k_{p}\left(T_{1}, \ldots, T_{e}\right)\right)=\mathrm{P} \Omega^{-}(2 m, q)$ for $2 \leq e \leq m-1$.

Consider the monic polynomials

$$
\phi^{-}=\phi^{-}(Y)=Y^{q^{2 m}-1}-1+\sum_{i=1}^{m-1}\left(T_{i}^{q^{i}} Y^{q^{m+i}-1}-T_{i} Y^{q^{m-i}-1}\right)
$$

and

$$
\phi_{e}^{-}=\phi_{e}^{-}(Y)=Y^{q^{2 m}-1}-1+\sum_{i=1}^{e}\left(T_{i}^{q^{i}} Y^{q^{m+i}-1}-T_{i} Y^{q^{m-i}-1}\right) \quad \text { for } 1 \leq e \leq m-1
$$

of degree $q^{2 m}-1$ in $Y$ with coefficients in $k_{p}\left[T_{1}, \ldots, T_{m-1}\right]$ and $k_{p}\left[T_{1}, \ldots, T_{e}\right]$, respectively, and note that, as before, $\operatorname{Disc}_{Y}\left(\phi^{-}\right)=\operatorname{Disc}_{Y}\left(\phi_{e}^{-}\right)=-1$. In Section 6, as a consequence of the above result about the Galois groups of $f^{-}$and $f_{e}^{-}$, we show that if $m>3 \leq p$ and $k_{p}$ is algebraically closed then $\operatorname{Gal}\left(\phi^{-}, k_{p}\left(T_{1}, \ldots, T_{m-1}\right)\right)=$ $\operatorname{Gal}\left(\phi_{e}^{-}, k_{p}\left(T_{1}, \ldots, T_{e}\right)\right)=\Omega^{-}(2 m, q)$ for $2 \leq e \leq m-1$.

In Section 5 , we give a review of linear algebra including definitions of $\mathrm{P}^{-}(2 m, q)$ and $\Omega^{-}(2 m, q)$.

\section{FACTORIZAtion of the Basic Equation}

We find a root $h_{m}(Y) \in \mathrm{GF}(p)[Y]$ of the polynomial

$$
Y^{q^{m}+1} R^{q}-R-\left(Y^{\langle 2 m-1\rangle}-1\right)
$$


by telescopically putting

$$
h_{m}(Y)=\sum_{\mu=0}^{m-1} Y^{\left(q^{m}+1\right)\langle m-2-\mu\rangle}
$$

and checking that then

$$
Y^{q^{m}+1} h_{m}(Y)^{q}-h_{m}(Y)-\left(Y^{\langle 2 m-1\rangle}-1\right)=0
$$

and, for any integer $0<i<m$, we find a root $h_{i}\left(Y, T_{i}\right) \in \mathrm{GF}(p)\left[Y, T_{i}\right]$ of the polynomial

$$
Y^{q^{m}+1} R^{q}-R-\left(T_{i}^{q^{i}} Y^{\langle m-1+i\rangle}-T_{i} Y^{\langle m-1-i\rangle}\right)
$$

by telescopically putting

$$
h_{i}\left(Y, T_{i}\right)=\sum_{\mu=0}^{i-1} T_{i}^{q^{i-1-\mu}} Y^{q^{m}\langle i-2-\mu\rangle+\langle m-2-\mu\rangle}
$$

and checking that then

$$
Y^{q^{m}+1} h_{i}\left(Y, T_{i}\right)^{q}-h_{i}\left(Y, T_{i}\right)-\left(T_{i}^{q^{i}} Y^{\langle m-1+i\rangle}-T_{i} Y^{\langle m-1-i\rangle}\right)=0 .
$$

By summing the above equations, upon letting

$$
\bar{f}=\bar{f}(Y)=\sum_{\mu=0}^{m-1} Y^{\left(q^{m}+1\right)\langle m-2-\mu\rangle}+\sum_{i=1}^{m-1} \sum_{\mu=0}^{i-1} T_{i}^{q^{i-1-\mu}} Y^{q^{m}\langle i-2-\mu\rangle+\langle m-2-\mu\rangle},
$$

we get

$$
Y^{q^{m}+1} \bar{f}(Y)^{q}-\bar{f}(Y)-f^{-}(Y)=0 .
$$

From the above equation it follows that

$$
f^{-}=\bar{f} f^{*} \quad \text { where } \quad f^{*}=f^{*}(Y)=Y^{q^{m}+1} \bar{f}(Y)^{q-1}-1
$$

and

$$
\text { if } p \neq 2 \text { then } f^{*}=f^{* *} f^{* * *}
$$

where

$$
f^{* *}=f^{* *}(Y)=Y^{\left(q^{m}+1\right) / 2} \bar{f}(Y)^{(q-1) / 2}-1
$$

and

$$
f^{* * *}=f^{* * *}(Y)=Y^{\left(q^{m}+1\right) / 2} \bar{f}(Y)^{(q-1) / 2}+1 .
$$

Note that the $(\mu=0)$ term in the above first summation is $Y^{\left(q^{m}+1\right)\langle m-2\rangle}$ and its exponent $\left(q^{m}+1\right)\langle m-2\rangle$ is strictly greater than the $Y$-exponent of every other term in the above two summations. Hence $\bar{f}$ is a monic polynomial of degree $\left(q^{m}+1\right)\langle m-2\rangle$ in $Y$ with coefficients in $k_{p}\left[T_{1}, \ldots, T_{m-1}\right]$. Therefore $f^{*}$ is a monic polynomial of degree $\left(q^{m}+1\right)[1+(q-1)\langle m-2\rangle]=q^{m-1}\left(q^{m}+1\right)$ in $Y$ with coefficients in $k_{p}\left[T_{1}, \ldots, T_{m-1}\right]$, and if $p \neq 2$ then $f^{* *}$ and $f^{* * *}$ are both monic 
polynomials of degree $q^{m-1}\left(q^{m}+1\right) / 2$ in $Y$ with coefficients in $k_{p}\left[T_{1}, \ldots, T_{m-1}\right]$. Thus

(3.0)

$f^{-}=\bar{f} f^{*}$ where $\bar{f}$ and $f^{*}$ are monic polynomials of degrees $\left(q^{m}+1\right)\langle m-2\rangle$

and $q^{m-1}\left(q^{m}+1\right)$ in $Y$ with coefficients in $k_{p}\left[T_{1}, \ldots, T_{m-1}\right]$ respectively,

and if $p \neq 2$ then $f^{*}=f^{* *} f^{* * *}$ where $f^{* *}$ and $f^{* * *}$ are both monic polynomials (of degree $q^{m-1}\left(q^{m}+1\right) / 2$ in $Y$ with coefficients in $k_{p}\left[T_{1}, \ldots, T_{m-1}\right]$.

For $1 \leq e \leq m-1$, let $\bar{f}_{e}=\bar{f}_{e}(Y)$ and $f_{e}^{*}=f_{e}^{*}(Y)$ be obtained by putting $T_{i}=0$ for all $i>e$ in $\bar{f}$ and $f^{*}$, respectively, and if $p \neq 2$ then let $f_{e}^{* *}=f_{e}^{* *}(Y)$ and $f_{e}^{* * *}=f_{e}^{* * *}(Y)$ be obtained by putting $T_{i}=0$ for all $i>e$ in $f^{* *}$ and $f^{* * *}$, respectively. Then by (3.0),

(3.1)

( for $1 \leq e \leq m-1$ we have:

$f_{e}^{-}=\bar{f}_{e} f_{e}^{*}$ where $\bar{f}_{e}$ and $f_{e}^{*}$ are monic polynomials of degrees $\left(q^{m}+1\right)\langle m-2\rangle$

and $q^{m-1}\left(q^{m}+1\right)$ in $Y$ with coefficients in $k_{p}\left[T_{1}, \ldots, T_{e}\right]$, respectively,

and if $p \neq 2$ then $f_{e}^{*}=f_{e}^{* *} f_{e}^{* * *}$ where $f_{e}^{* *}$ and $f_{e}^{* * *}$ are both monic polynomials of degree $q^{m-1}\left(q^{m}+1\right) / 2$ in $Y$ with coefficients in $k_{p}\left[T_{1}, \ldots, T_{e}\right]$.

Now

$$
f_{e}^{-}=A_{e} T_{1}^{q}-B_{e} T_{1}+C_{e}
$$

where

$$
0 \neq A_{e}=Y^{\langle m\rangle} \in k_{p}[Y] \text { and } 0 \neq B_{e}=Y^{\langle m-2\rangle} \in k_{p}[Y]
$$

and

$$
C_{e}=Y^{\langle 2 m-1\rangle}-1+\sum_{i=2}^{e}\left(T_{i}^{q^{i}} Y^{\langle m-1+i\rangle}-T_{i} Y^{\langle m-1-i\rangle}\right) \in k_{p}\left[Y, T_{1}, \ldots, T_{e}\right]
$$

and hence in particular $\operatorname{deg}_{T_{1}} f_{e}^{-}=q$. Also clearly $\operatorname{deg}_{T_{1}} \bar{f}_{e}=1$ and hence $\operatorname{deg}_{T_{1}} f_{e}^{*}=$ $q-1$ and if $p \neq 2$ then $\operatorname{deg}_{T_{1}} f_{e}^{* *}=(q-1) / 2=\operatorname{deg}_{T_{1}} f_{e}^{* * *}$.

In case of $p=2$, the irreducibility of $\bar{f}_{e}$ and $f_{e}^{*}$ will follow from Lemmas (4.2) and (4.3) of [A05]. In case of $p \neq 2$, for establishing the irreducibility of $\bar{f}_{e}, f_{e}^{* *}$ and $f_{e}^{* * *}$ we now prove the following lemma.

Lemma (3.2). Let $Q$ be a field of characteristic $p$ and consider a univariate polynomial $g_{0}=A_{0} T^{q}-B_{0} T+C_{0}$ with $A_{0}, B_{0}, C_{0}$ in $Q$ such that $A_{0} \neq 0 \neq B_{0}$. Assume that $g_{0}=g_{0}^{\prime} g_{0}^{\prime \prime} g_{0}^{\prime \prime \prime}$ in $Q[T]$ with $\operatorname{deg}_{T} g_{0}^{\prime}=1$ and $\operatorname{deg}_{T} g_{0}^{\prime \prime}>0<\operatorname{deg}_{T} g_{0}^{\prime \prime \prime}$. Also assume that for some real discrete valuation I of $Q$ (whose value group is the group of all integers) we have $G C D\left(q-1, I\left(B_{0} / A_{0}\right)\right)=2$. Then $g_{0}^{\prime \prime}$ and $g_{0}^{\prime \prime \prime}$ are irreducible in $Q[T]$.

To see this, we note that by assumption $g_{0}^{\prime}=A_{0}^{\prime} T+B_{0}^{\prime}$ with $0 \neq A_{0}^{\prime} \in Q$ and $B_{0}^{\prime} \in Q$. Now $-B_{0}^{\prime} / A_{0}^{\prime}$ is a root of $g_{0} / A_{0}=T^{q}-\left(B_{0} / A_{0}\right) T+\left(C_{0} / A_{0}\right)$ and hence

$$
\left[T-\left(B_{0}^{\prime} / A_{0}^{\prime}\right)\right]^{q}-\left(B_{0} / A_{0}\right)\left[T-\left(B_{0}^{\prime} / A_{0}^{\prime}\right)\right]+\left(C_{0} / A_{0}\right)=T\left[T^{q-1}-\left(B_{0} / A_{0}\right)\right] .
$$


Therefore, in view of the $Q$-automorphism $T \rightarrow T-\left(B_{0}^{\prime} / A_{0}^{\prime}\right)$ of $Q[T]$, we see that $g_{0} / A_{0}$ factors into exactly one more nonconstant monic irreducible factor in $Q[T]$ as $T^{q-1}-\left(B_{0} / A_{0}\right)$, i.e., upon writing $g_{0} / A_{0}=\theta_{1} \theta_{2} \ldots \theta_{\rho}$ and $T^{q-1}-$ $\left(B_{0} / A_{0}\right)=\theta_{1}^{\prime} \theta_{2}^{\prime} \ldots \theta_{\rho^{\prime}}^{\prime}$ where $\theta_{1}, \theta_{2}, \ldots, \theta_{\rho}, \theta_{1}^{\prime}, \theta_{2}^{\prime}, \ldots, \theta_{\rho^{\prime}}^{\prime}$ are nonconstant monic irreducible polynomials in $Q[t]$, we have $\rho=1+\rho^{\prime}$. By assumption 2 divides $q-1$ and hence we must have $p \neq 2$. Also 2 divides $I\left(B_{0} / A_{0}\right)$ and hence $I\left(B_{0} / A_{0}\right)=2 s$ where $s$ is an integer. We can take an element $\Lambda$ in $Q$ with $I(\Lambda)=1$, and then we can take an element $\Delta$ in an algebraic closure $Q^{*}$ of $Q$ with $B_{0} / A_{0}=\left(\Delta \Lambda^{s}\right)^{2}$. Now $I\left(\left(B_{0} / A_{0}\right) / \Lambda^{2 s}\right)=0$ and hence by the Discriminant Criterion we see that $I$ is unramified in $Q(\Delta)$. Therefore upon taking an extension $I^{*}$ of $I$ to $Q(\Delta)$ we have $I^{*}\left(\Delta \Lambda^{s}\right)=s$ and hence $\operatorname{GCD}\left((q-1) / 2, I^{*}\left(\Delta \Lambda^{s}\right)\right)=1=\operatorname{GCD}\left((q-1) / 2, I^{*}\left(-\Delta \Lambda^{s}\right)\right)$. In $Q(\Delta)[T]$ we have $T^{q-1}-\left(B_{0} / A_{0}\right)=\left[T^{(q-1) / 2}-\Delta \Lambda^{s}\right]\left[T^{(q-1) / 2}+\Delta \Lambda^{s}\right]$. By taking $\Delta^{\prime} \in Q^{*}$ with $\Delta^{\prime(q-1) / 2}=\Delta \Lambda^{s}$ and then taking an extension $I^{\prime}$ of $I^{*}$ to $Q\left(\Delta, \Delta^{\prime}\right)$ and letting $r$ be the reduced ramification exponent of $I^{\prime}$ over $I^{*}$, we have $I^{\prime}\left(\Delta \Lambda^{s}\right) /[(q-1) / 2]=r I^{*}\left(\Delta \Lambda^{s}\right) /[(q-1) / 2]=r s /[(q-1) / 2]$. Consequently $r s /[(q-1) / 2]$ must be an integer and hence, because $\operatorname{GCD}\left((q-1) / 2, I^{*}\left(\Delta \Lambda^{s}\right)\right)=1$, it follows that $r$ divides $(q-1) / 2$. Since the field degree $\left[Q\left(\Delta, \Delta^{\prime}\right): Q(\Delta)\right]$ is at least $r$, we conclude that $\left[Q\left(\Delta, \Delta^{\prime}\right): Q(\Delta)\right] \geq(q-1) / 2$. Since $\Delta^{\prime}$ is a root of the polynomial $T^{(q-1) / 2}-\Delta \Lambda^{s}$, this polynomial must be irreducible in $Q(\Delta)[T]$. Similarly the polynomial $T^{(q-1) / 2}+\Delta \Lambda^{s}$ is also irreducible in $Q(\Delta)[T]$. Consequently $\rho^{\prime} \leq 2$ and hence $\rho \leq 3$. Therefore the polynomials $g_{0}^{\prime \prime}$ and $g_{0}^{\prime \prime \prime}$ must be irreducible in $Q[T]$.

The following lemma is an easy consequence of the Gauss Lemma.

Lemma (3.3). Let $\kappa$ be a field, and let $g_{0}=g_{0}^{\prime} g_{0}^{\prime \prime} g_{0}^{\prime \prime \prime}$ where $g_{0}, g_{0}^{\prime}, g_{0}^{\prime \prime}, g_{0}^{\prime \prime \prime}$ are monic polynomials of positive degrees in $Z$ with coefficients in the $(d+1)$-variable polynomial ring $\kappa\left[X_{1}, \ldots, X_{d}, T\right]$. Assume that the polynomials $g_{0}^{\prime}, g_{0}^{\prime \prime}$, and $g_{0}^{\prime \prime \prime}$ have positive $T$-degrees and are irreducible in the ring $\kappa\left(X_{1}, \ldots, X_{d}, Z\right)[T]$. Also assume that the coefficients of $g_{0}$ as a polynomial in $T$ have no nonconstant common factor in $\kappa\left[X_{1}, \ldots, X_{d}, Z\right]$. Then the polynomials $g_{0}^{\prime}, g_{0}^{\prime \prime}$ and $g_{0}^{\prime \prime \prime}$ are irreducible in the ring $\kappa\left(X_{1}, \ldots, X_{d}, T\right)[Z]$.

By letting $I$ to be the $Y$-adic valuation of $Q=k_{p}\left(Y, T_{2}, \ldots, T_{e}\right)$, i.e., the real discrete valuation whose valuation ring is the localization of $k_{p}\left[Y, T_{2}, \ldots, T_{e}\right]$ at the principal prime ideal generated by $Y$, we see that $I\left(A_{e}\right)=\langle m\rangle$ and $I\left(B_{e}\right)=$ $\langle m-2\rangle$ and hence $I\left(B_{e} / A_{e}\right)=\langle m-2\rangle-\langle m\rangle=-q^{m-1}(1+q)$. Therefore $\operatorname{GCD}\left(q-1, I\left(B_{e} / A_{e}\right)\right)=1$ or 2 according as $p=2$ or $p \neq 2$. Also obviously $A_{e}$ and $C_{e}$ have no nonconstant common factors in $k_{p}\left[Y, T_{2}, \ldots, T_{e}\right]$. Therefore, if $p=2$ then by Lemmas (4.2) and (4.3) of [A05], and if $p \neq 2$ then by the above Lemmas (3.2) and (3.3), for $1 \leq e \leq m-1$ we have that

$$
\left\{\begin{array}{l}
\text { if } p=2 \text { then } \bar{f}_{e} \text { and } f_{e}^{*} \text { are irreducible in } k_{p}\left(T_{1}, \ldots, T_{e}\right)[Y], \text { and } \\
\text { if } p \neq 2 \text { then } \bar{f}_{e}, f_{e}^{* *} \text { and } f_{e}^{* * *} \text { are irreducible in } k_{p}\left(T_{1}, \ldots, T_{e}\right)[Y] .
\end{array}\right.
$$

By taking $e=m-1$ in (3.4) we see that

$$
\left\{\begin{array}{l}
\text { if } p=2 \text { then } \bar{f} \text { and } f^{*} \text { are irreducible in } k_{p}\left(T_{1}, \ldots, T_{m-1}\right)[Y], \text { and } \\
\text { if } p \neq 2 \text { then } \bar{f}, f^{* *} \text { and } f^{* * *} \text { are irreducible in } k_{p}\left(T_{1}, \ldots, T_{m-1}\right)[Y] .
\end{array}\right.
$$




\section{Twisted Derivative And its Factorization}

Recall that

$$
\bar{f}=\bar{f}(Y)=\sum_{\mu=0}^{m-1} Y^{\left(q^{m}+1\right)\langle m-2-\mu\rangle}+\sum_{i=1}^{m-1} \sum_{\mu=0}^{i-1} T_{i}^{q^{i-1-\mu}} Y^{q^{m}\langle i-2-\mu\rangle+\langle m-2-\mu\rangle} .
$$

Solving the equation $\bar{f}=0$, we get

$$
T_{1}=\frac{\sum_{\mu=0}^{m-1} Y^{\left(q^{m}+1\right)\langle m-2-\mu\rangle}+\sum_{i=2}^{m-1} \sum_{\mu=0}^{i-1} T_{i}^{q^{i-1-\mu}} Y^{q^{m}}\langle i-2-\mu\rangle+\langle m-2-\mu\rangle}{-Y^{\langle m-2\rangle}}
$$

and hence

$$
\begin{aligned}
& f^{\prime}(Y, Z)=\frac{\bar{f}(Z)-\bar{f}(Y)}{Z-Y} \quad\left(\text { def of the twisted derivative } f^{\prime} \text { of } \bar{f}\right) \\
& =\frac{\sum_{\mu=0}^{m-2}\left(Z^{\left(q^{m}+1\right)\langle m-2-\mu\rangle}-Y^{\left(q^{m}+1\right)\langle m-2-\mu\rangle}\right)}{Z-Y} \\
& +\frac{\sum_{\mu=0}^{m-1} Y^{\left(q^{m}+1\right)\langle m-2-\mu\rangle}}{-Y^{\langle m-2\rangle}} \times \frac{Z^{\langle m-2\rangle}-Y^{\langle m-2\rangle}}{Z-Y} \\
& +\frac{\sum_{i=2}^{m-1} \sum_{\mu=0}^{i-1} T_{i}^{q^{i-1-\mu}} Y^{q^{m}\langle i-2-\mu\rangle+\langle m-2-\mu\rangle}}{-Y^{\langle m-2\rangle}} \times \frac{Z^{\langle m-2\rangle}-Y^{\langle m-2\rangle}}{Z-Y} \\
& +\sum_{i=2}^{m-1} \sum_{\mu=0}^{i-1} \frac{T_{i}^{q^{i-1-\mu}}\left(Z^{q^{m}\langle i-2-\mu\rangle+\langle m-2-\mu\rangle}-Y^{q^{m}\langle i-2-\mu\rangle+\langle m-2-\mu\rangle}\right)}{Z-Y} .
\end{aligned}
$$

Therefore

$$
\begin{aligned}
g= & g(Y, Z) \\
= & Y^{\left(q^{m}+1\right)\langle m-2\rangle-1} f^{\prime}(1 / Y, Z / Y) \quad \begin{array}{c}
\text { def of polynomial } g \text { obtained by dividing } \\
\text { roots of } \left.f^{\prime} \text { by } Y \text { and then changing } Y \text { to } 1 / Y\right)
\end{array} \\
= & \frac{\sum_{\mu=0}^{m-2}\left(Z^{\left(q^{m}+1\right)\langle m-2-\mu\rangle}-1\right) Y^{\left(q^{m}+1\right) q^{m-1-\mu}\langle\mu-1\rangle}}{Z-1} \\
& +\frac{\sum_{\mu=0}^{m-1} Y^{\left(q^{m}+1\right) q^{m-1-\mu}\langle\mu-1\rangle}}{-1} \times \frac{Z^{\langle m-2\rangle}-1}{Z-1} \\
& +\frac{\sum_{i=2}^{m-1} \sum_{\mu=0}^{i-1} T_{i}^{q^{i-1-\mu}} Y^{q^{m-1-\mu+i}}\langle m-1+\mu-i\rangle+q^{m-1-\mu}\langle\mu-1\rangle}{-1} \times \frac{Z^{\langle m-2\rangle}-1}{Z-1} \\
& +\sum_{i=2}^{m-1} \sum_{\mu=0}^{i-1} \frac{T_{i}^{q^{i-1-\mu}}\left(Z^{q^{m}\langle i-2-\mu\rangle+\langle m-2-\mu\rangle}-1\right) Y^{q^{m-1-\mu+i}\langle m-1+\mu-i\rangle+q^{m-1-\mu}\langle\mu-1\rangle}}{Z-1} .
\end{aligned}
$$

For $i=m$, the powers of $Z$ in the last summation coincide with the corresponding powers of $Z$ in the first summation; moreover, for $\mu=m-1$, by convention $\left(Z^{\left(q^{m}+1\right)\langle m-2-\mu\rangle}-1\right)=0$, and hence the first summation can be extended to $m-1$. Consequently, upon letting

$$
D_{i \mu}=\frac{Z^{q^{m}\langle i-2-\mu\rangle+\langle m-2-\mu\rangle}-1}{Z-1}-\frac{Z^{\langle m-2\rangle}-1}{Z-1} \text { for } 2 \leq i \leq m \text { and } 0 \leq \mu \leq i-1
$$


we get

$$
\begin{aligned}
& g=\sum_{\mu=0}^{m-1} D_{m \mu} Y^{\left(q^{m}+1\right) q^{m-1-\mu}\langle\mu-1\rangle} \\
& +\sum_{i=2}^{m-1} \sum_{\mu=0}^{i-1} D_{i \mu} Y^{q^{m-1-\mu+i}\langle m-1+\mu-i\rangle+q^{m-1-\mu}\langle\mu-1\rangle} T_{i}^{q^{i-1-\mu}} .
\end{aligned}
$$

It follows that if $m=2$ then

$$
g=\frac{Z\left(Z^{q^{2}}-1\right)}{Z-1}-Y^{q^{2}+1} \quad \text { with } \quad \frac{Z\left(Z^{q^{2}}-1\right)}{Z-1} \in\left(Z k_{p}[Z]\right) \backslash\left(Z^{2} k_{p}[Z]\right)
$$

and hence $g$ is irreducible in $k_{p}(Z)[Y]$ and therefore by the Gauss Lemma $g$ is irreducible in $k_{p}(Y)[Z]$. Thus

$$
\left\{\begin{array}{l}
\text { if } m=2 \text {, then } g \text { is a monic polynomial of degree } q^{2} \text { in } Z \\
\text { with coefficients in } k_{p}[Y] \text {, and } g \text { is irreducible in } k_{p}(Y)[Z] .
\end{array}\right.
$$

Henceforth assuming $m>2$, and displaying dependence on $T_{2}$, we get

$$
\begin{aligned}
& g=D_{20} Y^{q^{m+1}}\langle m-3\rangle T_{2}^{q}+D_{21} Y^{q^{m}}\langle m-2\rangle+q^{m-2} T_{2} \\
& +\sum_{\mu=0}^{m-1} D_{m \mu} Y^{\left(q^{m}+1\right) q^{m-1-\mu}\langle\mu-1\rangle} \\
& +\sum_{i=3}^{m-1} \sum_{\mu=0}^{i-1} D_{i \mu} Y^{q^{m-1-\mu+i}}\langle m-1+\mu-i\rangle+q^{m-1-\mu}\langle\mu-1\rangle T_{i}^{q^{i-1-\mu}} .
\end{aligned}
$$

Now upon letting

$$
\widetilde{T}_{i}=Y^{q^{m}\langle m-1-i\rangle} T_{i} \quad \text { for } 2 \leq i \leq m-1
$$

we get

$$
\begin{aligned}
g= & D_{20} \widetilde{T}_{2}^{q}+D_{21} Y^{\left(q^{m}+1\right) q^{m-2}} \widetilde{T}_{2} \\
& +\sum_{\mu=0}^{m-1} D_{m \mu} Y^{\left(q^{m}+1\right) q^{m-1-\mu}\langle\mu-1\rangle} \\
& +\sum_{i=3}^{m-1} \sum_{\mu=0}^{i-1} D_{i \mu} Y^{\left(q^{m}+1\right) q^{m-1-\mu}\langle\mu-1\rangle} \widetilde{T}_{i}^{q^{i-1-\mu}} .
\end{aligned}
$$

Hence upon letting

$$
\widehat{Y}=Y^{q^{m}+1}
$$

and

$$
\widehat{T}_{i}= \begin{cases}\widetilde{T}_{i} & \text { for } 2 \leq i \leq m-1 \\ 1 & \text { for } i=m\end{cases}
$$


we get

$$
g=D_{20} \widehat{T}_{2}^{q}+D_{21} \widehat{Y}^{q^{m-2}} \widehat{T}_{2}+\sum_{i=3}^{m} \sum_{\mu=0}^{i-1} D_{i \mu} \widehat{Y}^{q^{m-1-\mu}\langle\mu-1\rangle} \widehat{T}_{i}^{q^{i-1-\mu}}
$$

Expanding the exponents of $\widehat{Y}$ we get

$$
g=D_{20} \widehat{T}_{2}^{q}+D_{21} \widehat{Y}^{q^{m-2}} \widehat{T}_{2}+\sum_{i=3}^{m} \sum_{\mu=0}^{i-1} D_{i \mu} \widehat{Y}^{q^{m-1-\mu}+\cdots+q^{m-2}} \widehat{T}_{i}^{q^{i-1-\mu}}
$$

where the dots indicate geometric series with ratio $q$. Upon letting

$$
\widehat{D}_{i \mu}=D_{i, i-1-\mu} \text { for } 2 \leq i \leq m \text { and } 0 \leq \mu \leq i-1
$$

we get

$$
\widehat{D}_{i \mu}=\frac{Z^{q^{m}\langle\mu-1\rangle+\langle m-1-i+\mu\rangle}-Z^{\langle m-2\rangle}}{Z-1} \text { for } 2 \leq i \leq m \text { and } 0 \leq \mu \leq i-1
$$

and arranging the terms according to descending powers of $\widehat{Y}$ we get

$$
g=\widehat{D}_{20} \widehat{Y}^{q^{m-2}} \widehat{T}_{2}+\widehat{D}_{21} \widehat{T}_{2}^{q}+\sum_{i=3}^{m} \sum_{\mu=0}^{i-1} \widehat{D}_{i \mu} \widehat{Y}^{q^{m-i+\mu}+\cdots+q^{m-2}} \widehat{T}_{i}^{q^{\mu}}
$$

and simplifying the expression of $\widehat{D}_{20}$ and $\widehat{D}_{21}$ we have

$$
\widehat{D}_{20}=-\frac{Z^{\langle m-3\rangle}\left(Z^{q^{m-2}}-1\right)}{Z-1} \quad \text { and } \quad \widehat{D}_{21}=\frac{Z^{\langle m-2\rangle}\left(Z^{q^{m}}-1\right)}{Z-1} .
$$

For a moment, assuming $m=3$, we note that

$$
g=\widehat{D}_{20} \widehat{Y}^{q} \widehat{T}_{2}+\widehat{D}_{21} \widehat{T}_{2}^{q}+\widehat{D}_{30} \widehat{Y}^{1+q}+\widehat{D}_{31} \widehat{Y}^{q}+\widehat{D}_{32}
$$

where

$$
\widehat{D}_{20}=-\frac{Z\left(Z^{q}-1\right)}{Z-1} \quad \text { and } \quad \widehat{D}_{21}=\frac{Z^{1+q}\left(Z^{q^{3}}-1\right)}{Z-1} \quad \text { and } \quad \widehat{D}_{30}=-\frac{\left(Z^{1+q}-1\right)}{Z-1}
$$

and

$$
\widehat{D}_{31}=\frac{Z^{1+q}\left(Z^{q^{3}-q}-1\right)}{Z-1} \quad \text { and } \quad \widehat{D}_{32}=\frac{Z^{1+q}\left(Z^{q^{3}+q^{4}}-1\right)}{Z-1}
$$

and to factor $g$ we try to find a $\widehat{T}_{2}$-root $E_{30} \widehat{Y}+E_{31}$ of $g$. To do this we first put

$$
E_{30}=\frac{\widehat{D}_{30}}{-\widehat{D}_{20}}=\frac{\frac{\left(Z^{1+q}-1\right)}{Z-1}}{\frac{-Z\left(Z^{q}-1\right)}{Z-1}}=\frac{\left(Z^{1+q}-1\right)}{-Z\left(Z^{q}-1\right)},
$$


then we put

$$
\begin{aligned}
E_{31} & =\frac{\widehat{D}_{31}+\widehat{D}_{21} E_{30}^{q}}{-\widehat{D}_{20}} \\
& =\frac{\frac{Z^{1+q}\left(Z^{q^{3}-q}-1\right)}{Z-1}+\frac{Z^{1+q}\left(Z^{q^{3}}-1\right)}{Z-1}\left(\frac{\left(Z^{1+q}-1\right)}{-Z\left(Z^{q}-1\right)}\right)^{q}}{\frac{Z\left(Z^{q}-1\right)}{Z-1}} \\
& =\frac{Z^{q}\left(Z^{q^{3}-q}-1\right)\left(Z^{q^{2}}-1\right)-\left(Z^{q^{3}}-1\right)\left(Z^{q^{2}+q}-1\right)}{\left(Z^{q}-1\right)\left(Z^{q^{2}}-1\right)} \\
& =\frac{\left(Z^{q^{3}+q^{2}}-Z^{q^{3}}-Z^{q^{2}+q}+Z^{q}\right)-\left(Z^{q^{3}+q^{2}+q}-Z^{q^{3}}-Z^{q^{2}+q}+1\right)}{\left(Z^{q}-1\right)\left(Z^{q^{2}}-1\right)} \\
& =\frac{Z^{q^{3}+q^{2}}-Z^{q^{3} q^{2}+q}+Z^{q}-1}{\left(Z^{q}-1\right)\left(Z^{q^{2}}-1\right)} \\
& =\frac{\left(Z^{q}-1\right)\left(-Z^{q^{3}+q^{2}}+1\right)}{\left(Z^{q}-1\right)\left(Z^{q^{2}}-1\right)} \\
& =\frac{\left(-Z^{q^{3}+q^{2}}+1\right)}{\left(Z^{q^{2}}-1\right)},
\end{aligned}
$$

and finally we calculate the term free of $\widehat{Y}$ to be

$$
\begin{aligned}
\widehat{D}_{32}+\widehat{D}_{21} E_{31}^{q} & =\frac{Z^{1+q}\left(Z^{q^{3}+q^{4}}-1\right)}{Z-1}+\left(\frac{Z^{1+q}\left(Z^{q^{3}}-1\right)}{Z-1}\right)\left(\frac{\left(-Z^{q^{3}+q^{2}}+1\right)}{\left(Z^{q^{2}}-1\right)}\right)^{q} \\
& =\frac{Z^{1+q}\left(Z^{q^{3}+q^{4}}-1\right)}{Z-1}+\frac{Z^{1+q}\left(-Z^{q^{3}+q^{4}}+1\right)}{Z-1} \\
& =0 .
\end{aligned}
$$

Alternatively, for "the fictitious term" $E_{32}$, we have

$$
\begin{aligned}
E_{32}=\frac{\widehat{D}_{32}+\widehat{D}_{21} E_{31}^{q}}{-\widehat{D}_{20}} & =\frac{\widehat{D}_{32}}{-\widehat{D}_{20}}+\left(\frac{\widehat{D}_{21}}{-\widehat{D}_{20}}\right)\left(\frac{\widehat{D}_{31}+\widehat{D}_{21} E_{30}^{q}}{-\widehat{D}_{20}}\right)^{q} \\
& =-\frac{\widehat{D}_{32}}{\widehat{D}_{20}}+\frac{\widehat{D}_{21} \widehat{D}_{31}^{q}}{\widehat{D}_{20}^{1+q}}+\frac{\widehat{D}_{21}^{1+q} E_{30}^{q^{2}}}{\widehat{D}_{20}^{1+q}} \\
& =-\frac{\widehat{D}_{32}}{\widehat{D}_{20}}+\frac{\widehat{D}_{21} \widehat{D}_{31}^{q}}{\widehat{D}_{20}^{1+q}}-\frac{\widehat{D}_{21}^{1+q} \widehat{D}_{30}^{q^{2}}}{\widehat{D}_{20}^{1+q+q^{2}}}
\end{aligned}
$$

and by substituting the values of $\widehat{D}_{20}, \widehat{D}_{21}, \widehat{D}_{30}, \widehat{D}_{31}, \widehat{D}_{32}$, we see this to be 0 .

Now, without assuming $m=3$, but henceforth again assuming $m>2$, to factor $g$, for any $3 \leq i \leq m$, we try to find a $\widehat{T}_{2}$-root

$$
\sum_{\mu=0}^{i-2} E_{i \mu} \widehat{Y}^{q^{m-i+\mu}+\cdots+q^{m-3}} \widehat{T}_{i}^{q^{\mu}}
$$


of

$$
\widehat{D}_{20} \widehat{Y}^{q^{m-2}} \widehat{T}_{2}+\widehat{D}_{21} \widehat{T}_{2}^{q}+\sum_{\mu=0}^{i-1} \widehat{D}_{i \mu} \widehat{Y}^{q^{m-i+\mu}+\cdots+q^{m-2}} \widehat{T}_{i}^{q^{\mu}}
$$

i.e., we try to find $E_{i \mu}$ in $\operatorname{GF}(p)(Z)$ such that

$$
\begin{aligned}
\sum_{\mu=0}^{i-1} \widehat{D}_{i \mu} \widehat{Y}^{q^{m-i+\mu}+\cdots+q^{m-2}} \widehat{T}_{i}^{q^{\mu}}= & -\widehat{D}_{20} \widehat{Y}^{q^{m-2}}\left(\sum_{\mu=0}^{i-2} E_{i \mu} \widehat{Y}^{q^{m-i+\mu}+\cdots+q^{m-3}} \widehat{T}_{i}^{q^{\mu}}\right) \\
& -\widehat{D}_{21}\left(\sum_{\mu=0}^{i-2} E_{i \mu} \widehat{Y}^{q^{m-i+\mu}+\cdots+q^{m-3}} \widehat{T}_{i}^{q^{\mu}}\right)^{q} .
\end{aligned}
$$

Equating coefficients of

$$
\widehat{Y}^{q^{m-i+\mu}+\cdots+q^{m-2}} \widehat{T}_{i}^{q^{\mu}}
$$

to zero, we try to find $E_{i \mu}$ in $\operatorname{GF}(p)(Z)$ such that

$$
\widehat{D}_{i \mu}= \begin{cases}-\widehat{D}_{20} E_{i \mu} & \text { for } \mu=0, \\ -\widehat{D}_{20} E_{i \mu}-\widehat{D}_{21} E_{i, \mu-1}^{q} & \text { for } 1 \leq \mu \leq i-2, \\ -\widehat{D}_{21} E_{i, \mu-1}^{q} & \text { for } \mu=i-1 .\end{cases}
$$

Since $\widehat{D}_{20} \neq 0$, we can successively find the values of $E_{i \mu}$ for $0 \leq \mu \leq i-2$ by solving all except the last equation, and then get a condition by substituting these in the last equation. Upon letting

$$
J_{i \mu}=\sum_{j=0}^{\mu}(-1)^{\langle\mu-j\rangle} \frac{\widehat{D}_{21}^{\langle\mu-j-1\rangle} \widehat{D}_{i j}^{q^{\mu-j}}}{\widehat{D}_{20}^{\langle\mu-j\rangle}} \quad \text { for } 0 \leq \mu \leq i-1
$$

these values are

$$
E_{i \mu}=J_{i \mu} \quad \text { for } 0 \leq \mu \leq i-2
$$

and the condition is

$$
J_{i, i-1}=0 \text {. }
$$

Substituting the simplified expressions of $\widehat{D}_{20}$ and $\widehat{D}_{21}$, for $0 \leq \mu \leq i-1$ and $0 \leq j \leq \mu$ we get

$$
\begin{aligned}
\frac{\widehat{D}_{21}^{\langle\mu-j-1\rangle}}{\widehat{D}_{20}^{\langle\mu-j\rangle}} & =\left[\frac{Z^{\langle m-2\rangle}\left(Z^{q^{m}}-1\right)}{Z-1}\right]^{\langle\mu-j-1\rangle}\left[\frac{Z-1}{-Z^{\langle m-3\rangle}\left(Z^{q^{m-2}}-1\right)}\right]^{\langle\mu-j\rangle} \\
& =\frac{Z^{\langle m-2\rangle\langle\mu-j-1\rangle-\langle m-3\rangle\langle\mu-j\rangle} \prod_{l=0}^{\mu-j-1}\left(Z^{q^{m}}-1\right)^{q^{l}}}{(-1)^{\langle\mu-j\rangle}(Z-1)^{-q^{\mu-j}} \prod_{l=0}^{\mu-j}\left(Z^{q^{m-2}}-1\right)^{q^{l}}} \\
& =\frac{Z^{\langle m-2\rangle\langle\mu-j-1\rangle-\langle m-3\rangle\langle\mu-j\rangle} \prod_{l=0}^{\mu-j-1}\left(Z^{q^{m+l}}-1\right)}{(-1)^{\langle\mu-j\rangle}(Z-1)^{-q^{\mu-j}} \prod_{l=0}^{\mu-j}\left(Z^{q^{m-2+l}}-1\right)} \\
& =\frac{Z^{\langle m-2\rangle\langle\mu-j-1\rangle-\langle m-3\rangle\langle\mu-j\rangle} \prod_{l=m}^{m+\mu-j-1}\left(Z^{q^{l}}-1\right)}{(-1)^{\langle\mu-j\rangle}(Z-1)^{-q^{\mu-j}} \prod_{l=m-2}^{m+\mu-j-2}\left(Z^{q^{l}}-1\right)} \\
& =\frac{Z^{\langle m-2\rangle\langle\mu-j-1\rangle-\langle m-3\rangle\langle\mu-j\rangle}\left(Z^{q^{m+\mu-j-1}}-1\right)}{(-1)^{\langle\mu-j\rangle}(Z-1)^{-q^{\mu-j}}\left(Z^{q^{m-2}}-1\right)\left(Z^{q^{m-1}}-1\right)}
\end{aligned}
$$


where, for the last equation, a separate but trivial argument may be made in the case of $j=\mu$ by noting that then the extra (purposefully inserted) term $\left(Z^{q^{m+\mu-j-1}}-1\right)$ in the numerator equals the extra term $\left(Z^{q^{m-1}}-1\right)$ in the denominator. Therefore by substituting the values of $\widehat{D}_{i j}$, for $0 \leq \mu \leq i-1$ we get

$$
\begin{aligned}
& J_{i \mu}= \sum_{j=0}^{\mu}(-1)^{\langle\mu-j\rangle}\left[\frac{Z^{\langle m-2\rangle\langle\mu-j-1\rangle-\langle m-3\rangle\langle\mu-j\rangle}\left(Z^{q^{m+\mu-j-1}}-1\right)}{(-1)^{\langle\mu-j\rangle}(Z-1)^{-q^{\mu-j}}\left(Z^{q^{m-2}}-1\right)\left(Z^{q^{m-1}}-1\right)}\right] \times \\
& \times\left[\frac{Z^{q^{m}\langle j-1\rangle+\langle m-1-i+j\rangle}-Z^{\langle m-2\rangle}}{Z-1}\right]^{q^{\mu-j}} \\
&=\sum_{j=0}^{\mu} \frac{Z^{\langle m-2\rangle\langle\mu-j-1\rangle-\langle m-3\rangle\langle\mu-j\rangle+q^{m+\mu-j}\langle j-1\rangle+q^{\mu-j}\langle m-1-i+j\rangle}\left(Z^{q^{m+\mu-j-1}}-1\right)}{\left(Z^{q^{m-2}}-1\right)\left(Z^{q^{m-1}}-1\right)} \\
&-\sum_{j=0}^{\mu} \frac{Z^{\langle m-2\rangle\langle\mu-j-1\rangle-\langle m-3\rangle\langle\mu-j\rangle+q^{\mu-j}\langle m-2\rangle}\left(Z^{q^{m+\mu-j-1}}-1\right)}{\left(Z^{q^{m-2}}-1\right)\left(Z^{q^{m-1}}-1\right)}
\end{aligned}
$$

where

the first exponent of $Z$ in the last summation

$$
\begin{aligned}
& =\langle m-2\rangle\langle\mu-j-1\rangle-\langle m-3\rangle\langle\mu-j\rangle+q^{\mu-j}\langle m-2\rangle \\
& =\left[\langle m-2\rangle\left(\langle\mu-j\rangle-q^{\mu-j}\right)-\left(\langle m-2\rangle-q^{m-2}\right)\langle\mu-j\rangle\right]+q^{\mu-j}\langle m-2\rangle \\
& =q^{m-2}\langle\mu-j\rangle
\end{aligned}
$$

and

the first exponent of $Z$ in the last but one summation

$$
\begin{aligned}
= & \langle m-2\rangle\langle\mu-j-1\rangle-\langle m-3\rangle\langle\mu-j\rangle+q^{m+\mu-j}\langle j-1\rangle+q^{\mu-j}\langle m-1-i+j\rangle \\
= & {\left[\langle m-2\rangle\left(\langle\mu-j\rangle-q^{\mu-j}\right)-\left(\langle m-2\rangle-q^{m-2}\right)\langle\mu-j\rangle\right] } \\
& \quad+q^{m+\mu-j}\langle j-1\rangle+q^{\mu-j}\langle m-1-i+j\rangle \\
= & {\left[q^{m-2}\langle\mu-j\rangle-q^{\mu-j}\langle m-2\rangle\right]+q^{m+\mu-j}\langle j-1\rangle+q^{\mu-j}\langle m-1-i+j\rangle } \\
= & {\left[q^{m-2}\langle\mu-j\rangle+q^{m+\mu-j-1}+q^{m+\mu-j}\langle j-1\rangle\right]-q^{\mu-j}[\langle m-2\rangle-\langle m-1-i+j\rangle] } \\
& \quad-q^{m+\mu-j-1} \\
= & q^{m-2}\langle\mu+1\rangle-q^{m+\mu-i}\langle i-2-j\rangle-q^{m+\mu-j-1} .
\end{aligned}
$$


Hence

$$
\begin{aligned}
& J_{i \mu}=\sum_{j=0}^{\mu} \frac{Z^{q^{m-2}\langle\mu+1\rangle-q^{m+\mu-i}\langle i-2-j\rangle-q^{m+\mu-j-1}}\left(Z^{q^{m+\mu-j-1}}-1\right)}{\left(Z^{q^{m-2}}-1\right)\left(Z^{q^{m-1}}-1\right)} \\
& -\sum_{j=0}^{\mu} \frac{Z^{q^{m-2}\langle\mu-j\rangle}\left(Z^{q^{m+\mu-j-1}}-1\right)}{\left(Z^{q^{m-2}}-1\right)\left(Z^{q^{m-1}}-1\right)} \\
& =\sum_{j=0}^{\mu} \frac{Z^{q^{m-2}\langle\mu+1\rangle-q^{m+\mu-i}\langle i-2-j\rangle}}{\left(Z^{q^{m-2}}-1\right)\left(Z^{q^{m-1}}-1\right)}-\sum_{j=0}^{\mu} \frac{Z^{q^{m-2}\langle\mu+1\rangle-q^{m+\mu-i}\langle i-1-j\rangle}}{\left(Z^{q^{m-2}}-1\right)\left(Z^{q^{m-1}}-1\right)} \\
& -\sum_{j=0}^{\mu} \frac{Z^{q^{m-2}}\langle\mu-j+1\rangle}{\left(Z^{q^{m-2}}-1\right)\left(Z^{q^{m-1}}-1\right)}+\sum_{j=0}^{\mu} \frac{Z^{q^{m-2}}\langle\mu-j\rangle}{\left(Z^{q^{m-2}}-1\right)\left(Z^{q^{m-1}}-1\right)} \\
& =\sum_{j=1}^{\mu+1} \frac{Z^{q^{m-2}\langle\mu+1\rangle-q^{m+\mu-i}\langle i-1-j\rangle}}{\left(Z^{q^{m-2}}-1\right)\left(Z^{q^{m-1}}-1\right)}-\sum_{j=0}^{\mu} \frac{Z^{q^{m-2}}\langle\mu+1\rangle-q^{m+\mu-i}\langle i-1-j\rangle}{\left(Z^{q^{m-2}}-1\right)\left(Z^{q^{m-1}}-1\right)} \\
& -\sum_{j=0}^{\mu} \frac{Z^{q^{m-2}\langle\mu-j+1\rangle}}{\left(Z^{q^{m-2}}-1\right)\left(Z^{q^{m-1}}-1\right)}+\sum_{j=1}^{\mu+1} \frac{Z^{q^{m-2}}\langle\mu-j+1\rangle}{\left(Z^{q^{m-2}}-1\right)\left(Z^{q^{m-1}}-1\right)} \\
& =\frac{Z^{q^{m-2}\langle\mu+1\rangle-q^{m+\mu-i}\langle i-2-\mu\rangle}}{\left(Z^{q^{m-2}}-1\right)\left(Z^{q^{m-1}}-1\right)}-\frac{Z^{q^{m-2}\langle\mu+1\rangle-q^{m+\mu-i}\langle i-1\rangle}}{\left(Z^{q^{m-2}}-1\right)\left(Z^{q^{m-1}}-1\right)} \\
& -\frac{Z^{q^{m-2}\langle\mu+1\rangle}}{\left(Z^{q^{m-2}}-1\right)\left(Z^{q^{m-1}}-1\right)}+\frac{Z^{q^{m-2}}}{\left(Z^{q^{m-2}}-1\right)\left(Z^{q^{m-1}}-1\right)} \\
& =\frac{Z^{q^{m-2}\langle\mu+1\rangle-q^{m+\mu-i}\langle i-1\rangle}\left(Z^{q^{m+\mu-i}(\langle i-1\rangle-\langle i-2-\mu\rangle)}-1\right)}{\left(Z^{q^{m-2}}-1\right)\left(Z^{q^{m-1}}-1\right)} \\
& -\frac{Z^{q^{m-2}}\left(Z^{q^{m-2}(\langle\mu+1\rangle-1)}-1\right)}{\left(Z^{q^{m-2}}-1\right)\left(Z^{q^{m-1}}-1\right)} \\
& =\frac{\left(Z^{q^{m-2}\langle\mu+1\rangle-q^{m+\mu-i}\langle i-1\rangle}-Z^{q^{m-2}}\right)\left(Z^{q^{m-1}\langle\mu\rangle}-1\right)}{\left(Z^{q^{m-2}}-1\right)\left(Z^{q^{m-1}}-1\right)} .
\end{aligned}
$$

Therefore

$$
\begin{aligned}
& J_{i \mu}=\frac{\left(Z^{-q^{m+\mu-i}\langle i-3-\mu\rangle}-Z^{q^{m-2}}\right)\left(Z^{q^{m-1}\langle\mu\rangle}-1\right)}{\left(Z^{q^{m-2}}-1\right)\left(Z^{q^{m-1}}-1\right)} \\
& =\frac{-\left(Z^{q^{m+\mu-i}\langle i-2-\mu\rangle}-1\right)\left(Z^{q^{m-1}}\langle\mu\rangle-1\right)}{Z^{q^{m+\mu-i}\langle i-3-\mu\rangle}\left(Z^{q^{m-2}}-1\right)\left(Z^{q^{m-1}}-1\right)} .
\end{aligned}
$$

Now by putting $\mu=i-1$ we see that

$$
J_{i, i-1}=0 .
$$

It follows that, upon letting

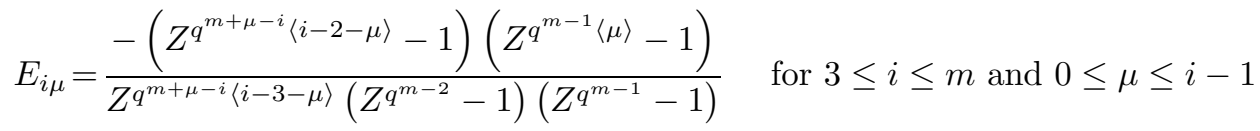


we have

$$
\begin{aligned}
\sum_{\mu=0}^{i-1} \widehat{D}_{i \mu} \widehat{Y}^{q^{m-i+\mu}+\cdots+q^{m-2}} \widehat{T}_{i}^{q^{\mu}} & \\
= & -\widehat{D}_{20} \widehat{Y}^{q^{m-2}}\left(\sum_{\mu=0}^{i-2} E_{i \mu} \widehat{Y}^{q^{m-i+\mu}+\cdots+q^{m-3}} \widehat{T}_{i}^{q^{\mu}}\right) \\
& -\widehat{D}_{21}\left(\sum_{\mu=0}^{i-2} E_{i \mu} \widehat{Y}^{q^{m-i+\mu}+\cdots+q^{m-3}} \widehat{T}_{i}^{\mu^{\mu}}\right)^{q} \text { for } 3 \leq i \leq m .
\end{aligned}
$$

By $q$-linearity, summing the above equations we get

$$
\begin{aligned}
\sum_{i=3}^{m} \sum_{\mu=0}^{i-1} \widehat{D}_{i \mu} \widehat{Y}^{q^{m-i+\mu}+\cdots+q^{m-2}} \widehat{T}_{i}^{q^{\mu}} & \\
= & -\widehat{D}_{20} \widehat{Y}^{q^{m-2}}\left(\sum_{i=3}^{m} \sum_{\mu=0}^{i-2} E_{i \mu} \widehat{Y}^{q^{m-i+\mu}+\cdots+q^{m-3}} \widehat{T}_{i}^{q^{\mu}}\right) \\
& -\widehat{D}_{21}\left(\sum_{i=3}^{m} \sum_{\mu=0}^{i-2} E_{i \mu} \widehat{Y}^{q^{m-i+\mu}+\cdots+q^{m-3}} \widehat{T}_{i}^{q^{\mu}}\right)^{q} .
\end{aligned}
$$

Therefore recalling that

$$
\widehat{D}_{20}=-\frac{Z^{\langle m-3\rangle}\left(Z^{q^{m-2}}-1\right)}{Z-1} \quad \text { and } \quad \widehat{D}_{21}=\frac{Z^{\langle m-2\rangle}\left(Z^{q^{m}}-1\right)}{Z-1}
$$

and letting

$$
D=-\widehat{D}_{21} / \widehat{D}_{20}^{q} \quad \text { and } \quad E=\widehat{D}_{20} \sum_{i=3}^{m} \sum_{\mu=0}^{i-2} E_{i \mu} \widehat{Y}^{q^{m-i+\mu}+\cdots+q^{m-3}} \widehat{T}_{i}^{q^{\mu}}
$$

we get

$$
D=Z(Z-1)^{\left(q^{m-1}+1\right)(q-1)}
$$

and

$E=\sum_{i=3}^{m} \sum_{\mu=0}^{i-2}\left(\frac{Z^{q^{m+\mu-i}\langle i-2-\mu\rangle}-1}{Z-1}\right)\left(\frac{Z^{\langle\mu\rangle}-1}{Z-1}\right)^{q^{m-1}} Z^{\langle m+\mu-i-1\rangle} \widehat{Y}^{q^{m-i+\mu}+\cdots+q^{m-3}} \widehat{T}_{i}^{q^{\mu}}$

and

$$
-D E^{q}+\widehat{Y}^{q^{m-2}} E+\sum_{i=3}^{m} \sum_{\mu=0}^{i-1} \widehat{D}_{i \mu} \widehat{Y}^{q^{m-i+\mu}+\cdots+q^{m-2}} \widehat{T}_{i}^{q^{\mu}}=0
$$

The above equation says that $E / \widehat{D}_{20}$ is a $\widehat{T}_{2}$-root of

$$
g=\widehat{D}_{21} \widehat{T}_{2}^{q}+\widehat{D}_{20} \widehat{Y}^{q^{m-2}} \widehat{T}_{2}+\sum_{i=3}^{m} \sum_{\mu=0}^{i-1} \widehat{D}_{i \mu} \widehat{Y}^{q^{m-i+\mu}+\cdots+q^{m-2}} \widehat{T}_{i}^{q^{\mu}}
$$


Hence upon letting

$$
g^{\prime}=E-\widehat{D}_{20} \widehat{T}_{2} \quad \text { and } \quad g^{\prime \prime}=D E^{q-1}-\widehat{Y}^{q^{m-2}}+\sum_{l=1}^{q-1} D \widehat{D}_{20}^{l} E^{q-1-l} \widehat{T}_{2}^{l}
$$

we obtain

$$
\begin{aligned}
g^{\prime} g^{\prime \prime}= & \left(D E^{q}-\widehat{Y}^{q^{m-2}} E\right)+\sum_{l=1}^{q-1} D \widehat{D}_{20}^{l} E^{q-l} \widehat{T}_{2}^{l} \\
& -\left(D \widehat{D}_{20} E^{q-1}-\widehat{D}_{20} \widehat{Y}^{q^{m-2}}\right) \widehat{T}_{2}-\sum_{l=2}^{q} D \widehat{D}_{20}^{l} E^{q-l} \widehat{T}_{2}^{l} \\
= & \left(D E^{q}-\widehat{Y}^{q^{m-2}} E\right)+\left(D \widehat{D}_{20} E^{q-1}\right) \widehat{T}_{2}+\sum_{l=2}^{q-1} D \widehat{D}_{20}^{l} E^{q-l} \widehat{T}_{2}^{l} \\
& -\left(D \widehat{D}_{20} E^{q-1}-\widehat{D}_{20} \widehat{Y}^{q^{m-2}}\right) \widehat{T}_{2}-\left(\sum_{l=2}^{q-1} D \widehat{D}_{20}^{l} E^{q-l} \widehat{T}_{2}^{l}\right)-D \widehat{D}_{20}^{q} \widehat{T}_{2}^{q} \\
= & \widehat{D}_{21} \widehat{T}_{2}^{q}+\widehat{D}_{20} \widehat{Y}^{q^{m-2}} \widehat{T}_{2}+\left(D E^{q}-\widehat{Y}^{q^{m-2}} E\right) \\
= & \widehat{D}_{21} \widehat{T}_{2}^{q}+\widehat{D}_{20} \widehat{Y}^{q^{m-2}} \widehat{T}_{2}+\sum_{i=3}^{m} \sum_{\mu=0}^{i-1} \widehat{D}_{i \mu} \widehat{Y}^{q^{m-i+\mu}+\cdots+q^{m-2}} \widehat{T}_{i}^{q^{\mu}} \\
= & g .
\end{aligned}
$$

Thus we get the factorization

$$
g=g^{\prime} g^{\prime \prime}
$$

where by substituting the values of $\widehat{Y}$ and $\widehat{T}_{i}$ we have

(4.2)

$$
\begin{aligned}
g= & \widehat{D}_{21} Y^{q^{m+1}\langle m-3\rangle} T_{2}^{q}+\widehat{D}_{20} \widehat{Y}^{q^{m-2}+q^{m}\langle m-2\rangle} T_{2}+\sum_{\mu=0}^{m-1} \widehat{D}_{m \mu} Y^{\left(q^{m}+1\right) q^{\mu}\langle m-2-\mu\rangle} \\
& +\sum_{i=3}^{m-1} \sum_{\mu=0}^{i-1} \widehat{D}_{i \mu} Y^{\left(q^{m}+1\right) q^{m-i+\mu}\langle i-2-\mu\rangle+q^{m+\mu}\langle m-1-i\rangle} T_{i}^{q^{\mu}}
\end{aligned}
$$

and

$$
g^{\prime}=E-\widehat{D}_{20} Y^{q^{m}\langle m-3\rangle} T_{2}
$$

and

$$
g^{\prime \prime}=D E^{q-1}-Y^{\left(q^{m}+1\right) q^{m-2}}+\sum_{l=1}^{q-1} D \widehat{D}_{20}^{l} E^{q-1-l} Y^{q^{m}\langle m-3\rangle l} T_{2}^{l}
$$

and

(4.5)

$E=\sum_{\mu=0}^{m-2} \widehat{E}_{m \mu} Y^{\left(q^{m}+1\right) q^{\mu}\langle m-3-\mu\rangle}+\sum_{i=3}^{m-1} \sum_{\mu=0}^{i-2} \widehat{E}_{i \mu} Y^{\left(q^{m}+1\right) q^{m-i+\mu}\langle i-3-\mu\rangle+q^{m+\mu}\langle m-1-i\rangle} T_{i}^{q^{\mu}}$ 
with

$$
\begin{gathered}
\widehat{E}_{i \mu}=\left(\frac{Z^{q^{m+\mu-i}\langle i-2-\mu\rangle}-1}{Z-1}\right)\left(\frac{Z^{\langle\mu\rangle}-1}{Z-1}\right)^{q^{m-1}} Z^{\langle m+\mu-i-1\rangle} \\
\text { for } 3 \leq i \leq m \text { and } 0 \leq \mu \leq i-2,
\end{gathered}
$$

and where we recall that

$$
\widehat{D}_{i \mu}=\frac{Z^{q^{m}\langle\mu-1\rangle+\langle m-1-i+\mu\rangle}-Z^{\langle m-2\rangle}}{Z-1} \quad \text { for } 3 \leq i \leq m \text { and } 0 \leq \mu \leq i-1
$$

and

$$
\widehat{D}_{20}=-\frac{Z^{\langle m-3\rangle}\left(Z^{q^{m-2}}-1\right)}{Z-1} \text { and } \widehat{D}_{21}=\frac{Z^{\langle m-2\rangle}\left(Z^{q^{m}}-1\right)}{Z-1}
$$

and

$$
D=-\widehat{D}_{21} / \widehat{D}_{20}^{q}=Z(Z-1)^{\left(q^{m-1}+1\right)(q-1)}
$$

By (4.6) we see that, for $3 \leq i \leq m$ and $0 \leq \mu \leq i-2, \widehat{E}_{i \mu}$ is a monic polynomial of degree

$q^{m+\mu-i}\langle i-2-\mu\rangle-1+q^{m-1}(\langle\mu\rangle-1)+\langle m+\mu-i-1\rangle=q\langle m-3\rangle+q^{m}\langle\mu-1\rangle$

in $Z$ with coefficients on $\operatorname{GF}(p)$. Therefore, since $Y^{\left(q^{m}+1\right) q^{\mu}\langle m-3-\mu\rangle}=1$ for $\mu=$ $m-2$, by $(4.5)$ we see that $E$ is a monic polynomial of degree

$$
q\langle m-3\rangle+q^{m}\langle(m-2)-1\rangle=q\left(q^{m-1}+1\right)\langle m-3\rangle
$$

in $Z$ with coefficients in $\operatorname{GF}(p)\left[Y, T_{2}, \ldots, T_{m-1}\right]$. Consequently, in view of (4.3) and (4.8) we conclude that $g^{\prime}$ is a monic polynomial of degree $q\left(q^{m-1}+1\right)\langle m-3\rangle$ in $Z$ with coefficients in $\operatorname{GF}(p)\left[Y, T_{2}, \ldots, T_{m-1}\right]$. Obviously $g$ is a monic polynomial of degree

$$
\left(\operatorname{deg}_{Y} \bar{f}\right)-1=\left(q^{m}+1\right)\langle m-2\rangle-1=q^{m}\langle m-2\rangle+q\langle m-3\rangle
$$

in $Z$ with coefficients in $\operatorname{GF}(p)\left[Y, T_{2}, \ldots, T_{m-1}\right]$. Hence in view of (4.1), (4.4), (4.8) and (4.9) we see that $g^{\prime \prime}$ is a monic polynomial of degree

$$
q^{m}\langle m-2\rangle+q\langle m-3\rangle-q\left(q^{m-1}+1\right)\langle m-3\rangle=q^{2 m-2}
$$

in $Z$ with coefficients in $\operatorname{GF}(p)\left[Y, T_{2}, \ldots, T_{m-1}\right]$. Thus

(4.10)

$\left\{g^{\prime}\right.$ and $g^{\prime \prime}$ are monic polynomials of degrees $q\left(q^{m-1}+1\right)\langle m-3\rangle$ and $q^{2 m-2}$

\{ in $Z$ with coefficients in $\operatorname{GF}(p)\left[Y, T_{2}, \ldots, T_{m-1}\right]$ respectively.

Without assuming $m>2$, for $1 \leq e \leq m-1$, let $f_{e}^{\prime}$ and $g_{e}$ denote the members of $\operatorname{GF}(p)\left[Y, Z, T_{2}, \ldots, T_{e}\right]$ obtained by putting $T_{i}=0$ for all $i>e$ in $f^{\prime}$ and $g$ 
respectively. Then $f_{e}^{\prime}$ is the twisted derivative of $\bar{f}_{e}$, and dividing the $Z$-roots of $f_{e}^{\prime}$ by $Y$ and afterwards changing $Y$ to $1 / Y$ we get $g_{e}$ which is a monic polynomial of degree $q^{m}\langle m-2\rangle+q\langle m-3\rangle$ in $Z$ with coefficients in $\operatorname{GF}(p)\left[Y, T_{2}, \ldots, T_{e}\right]$.

Again henceforth assuming $m>2$, for $1 \leq e \leq m-1$, let $g_{e}^{\prime}$ and $g_{e}^{\prime \prime}$ denote the members of $\operatorname{GF}(p)\left[Y, Z, T_{2}, \ldots, T_{e}\right]$ obtained by putting $T_{i}=0$ for all $i>e$ in $g^{\prime}$ and $g^{\prime \prime}$ respectively. Then in view of (4.1) and (4.10),

$$
\left\{\begin{array}{l}
\text { for } 1 \leq e \leq m-1 \text { we have } g_{e}=g_{e}^{\prime} g_{e}^{\prime \prime} \text { where } g_{e}^{\prime} \text { and } g_{e}^{\prime \prime} \text { are } \\
\text { monic polynomials of degrees } q\left(q^{m-1}+1\right)\langle m-3\rangle \text { and } q^{2 m-2} \text { in } Z \\
\text { with coefficients in } \operatorname{GF}(p)\left[Y, T_{2}, \ldots, T_{e}\right] \text { respectively. }
\end{array}\right.
$$

By (4.2), (4.3), (4.5), (4.6), (4.7) and (4.8) we have

$$
g_{2}=A_{2} T_{2}^{q}-B_{2} T_{2}+C_{2} \quad \text { and } \quad g_{2}^{\prime}=A_{2}^{\prime} T_{2}+B_{2}^{\prime}
$$

where $A_{2}, B_{2}, C_{2}, A_{2}^{\prime}, B_{2}^{\prime}$ are the nonzero elements in $\operatorname{GF}(p)[Y, Z]$ given by

$$
A_{2}=\widehat{D}_{21} Y^{q^{m+1}}\langle m-3\rangle \quad \text { and } \quad B_{2}=-\widehat{D}_{20} \widehat{Y}^{q^{m-2}+q^{m}\langle m-2\rangle}
$$

and

$$
C_{2}=\sum_{\mu=0}^{m-1} \widehat{D}_{m \mu} Y^{\left(q^{m}+1\right) q^{\mu}\langle m-2-\mu\rangle}
$$

and

$$
A_{2}^{\prime}=-\widehat{D}_{20} Y^{q^{m}\langle m-3\rangle} \quad \text { and } \quad B_{2}^{\prime}=\sum_{\mu=0}^{m-2} \widehat{E}_{m \mu} Y^{\left(q^{m}+1\right) q^{\mu}\langle m-3-\mu\rangle} .
$$

By letting $I$ to be the $Z$-adic valuation of $Q=k_{p}(Y, Z)$, i.e., the real discrete valuation whose valuation ring is the localization of $k_{p}[Y, Z]$ at the principal prime ideal generated by $Z$, we see that $I\left(A_{2}\right)=\langle m-2\rangle$ and $I\left(B_{2}\right)=\langle m-3\rangle$ and hence $I\left(B_{2} / A_{2}\right)=\langle m-3\rangle-\langle m-2\rangle=-q^{m-2}$ and therefore $\operatorname{GCD}\left(q-1, I\left(B_{2} / A_{2}\right)\right)=1$. In view of (4.7) and (4.8) we also see that $A_{2}$ and $C_{2}$ have no nonconstant common factor in $k_{p}[Y, Z]$, because $\mu=m-1$ gives the nonzero term $\widehat{D}_{m, m-1}$ of $C_{2}$ which is independent of $Y$, and $\mu=0$ gives the highest $Y$-degree term of $C_{2}$ and its coefficient is

$$
\widehat{D}_{m 0}=\frac{1-Z^{\langle m-2\rangle}}{Z-1} .
$$

Therefore by Lemmas (4.2) and (4.3) of [A05] we conclude that

$$
\text { the polynomials } g_{2}^{\prime} \text { and } g_{2}^{\prime \prime} \text { are irreducible in } k_{p}\left(Y, T_{2}\right)[Z] \text {. }
$$

As an immediate consequence of (4.12) we see that

$$
\left\{\begin{array}{l}
\text { the polynomials } g^{\prime} \text { and } g^{\prime \prime} \text { are irreducible in } k_{p}\left(Y, T_{2}, \ldots, T_{m-1}\right)[Z] \\
\text { and, for } 2 \leq e \leq m-1, \\
\text { the polynomials } g_{e}^{\prime} \text { and } g_{e}^{\prime \prime} \text { are irreducible in } k_{p}\left(Y, T_{2}, \ldots, T_{e}\right)[Z]
\end{array}\right.
$$

Note that

$$
\text { in (4.1) to (4.13) we assumed } m>2 \text {. }
$$

Recall that $\bar{f}_{e}$ is irreducible in $k_{p}\left(T_{1}, T_{2}, \ldots, T_{e}\right)[Y]$, its twisted derivative is $f_{e}^{\prime}(Y, Z)$, and $g_{e}$ is obtained by dividing the $Z$-roots of $f_{e}^{\prime}(Y, Z)$ by $Y$ and then changing $Y$ to $1 / Y$; therefore by (4.0), (4.1), (4.10), (4.11), (4.13) and (4.14) we get the following 
Theorem (4.15). If $m=2$ then $\operatorname{Gal}\left(\bar{f}, k_{p}\left(T_{1}\right)\right)=\operatorname{Gal}\left(\bar{f}_{1}, k_{p}\left(T_{1}\right)\right)$ is a 2-transitive permutation group of degree $q^{m}+1$. If $m>2$ and $2 \leq e \leq m-1$ then $\operatorname{Gal}\left(\bar{f}_{e}, k_{p}\left(T_{1}, \ldots, T_{e}\right)\right)$ is a transitive permutation group of Rank 3 with subdegrees $1, q\left(q^{m-1}+1\right)\langle m-3\rangle$ and $q^{2 m-2}$. Hence in particular, if $m>2$ then $\operatorname{Gal}\left(\bar{f}, k_{p}\left(T_{1}, \ldots, T_{m-1}\right)\right)$ is a transitive permutation group of Rank 3 with subdegrees $1, q\left(q^{m-1}+1\right)\langle m-3\rangle$ and $q^{2 m-2}$.

Notation. Recall that $<$ denotes a subgroup, and $\triangleleft$ denotes a normal subgroup. Let the groups $\mathrm{SL}(m, q) \triangleleft \mathrm{GL}(m, q) \triangleleft \Gamma \mathrm{L}(m, q)$ and $\mathrm{PSL}(m, q) \triangleleft \mathrm{PGL}(m, q) \triangleleft \mathrm{P} \Gamma \mathrm{L}(m, q)$ and their actions on $\operatorname{GF}(q)^{m}$ and $\mathcal{P}\left(\mathrm{GF}(q)^{m}\right)$ be as on pages 78-80 of [A03]. Let

$$
\Theta_{m}: \Gamma \mathrm{L}(m, q) \rightarrow \operatorname{P\Gamma L}(m, q)=\Gamma \mathrm{L}(m, q) / \mathrm{GF}(q)^{*}
$$

be the canonical epimorphism where we identify the multiplicative group $\operatorname{GF}(q)^{*}$ with scalar matrices, which constitute the center of $\operatorname{GL}(m, q)$.

Now in view of Proposition 3.1 of [A04], by (3.0), (3.1), (3.4) and (3.5) we get the following

Theorem (4.16). Assuming $G F(q) \subset k_{p}$, for $1 \leq e \leq m-1$, in a natural manner we may regard

$$
\operatorname{Gal}\left(\phi_{e}^{-}, k_{p}\left(T_{1}, \ldots, T_{e}\right)\right)<G L(2 m, q) \text { and } \operatorname{Gal}\left(f_{e}^{-}, k_{p}\left(T_{1}, \ldots, T_{e}\right)\right)<P G L(2 m, q)
$$

and then

$$
\Theta_{2 m}\left(\operatorname{Gal}\left(\phi_{e}^{-}, k_{p}\left(T_{1}, \ldots, T_{e}\right)\right)\right)=\operatorname{Gal}\left(f_{e}^{-}, k_{p}\left(T_{1}, \ldots, T_{e}\right)\right)
$$

and $\operatorname{Gal}\left(f_{e}^{-}, k_{p}\left(T_{1}, \ldots, T_{e}\right)\right)$ has two or three orbits on $\mathcal{P}\left(G F(q)^{2 m}\right)$ of sizes $\left(q^{m}+1\right)\langle m-2\rangle, q^{m-1}\left(q^{m}+1\right)$ or $\left(q^{m}+1\right)\langle m-2\rangle, q^{m-1}\left(q^{m}+1\right) / 2$, $q^{m-1}\left(q^{m}+1\right) / 2$ according as $p=2$ or $p \neq 2$. In particular, again assuming $G F(q) \subset k_{p}$, in a natural manner we may regard

$$
\operatorname{Gal}\left(\phi^{-}, k_{p}\left(T_{1}, \ldots, T_{m-1}\right)\right)<G L(2 m, q)
$$

and

$$
\operatorname{Gal}\left(f^{-}, k_{p}\left(T_{1}, \ldots, T_{m-1}\right)\right)<P G L(2 m, q)
$$

and then

$$
\Theta_{2 m}\left(\operatorname{Gal}\left(\phi^{-}, k_{p}\left(T_{1}, \ldots, T_{m-1}\right)\right)\right)=\operatorname{Gal}\left(f^{-}, k_{p}\left(T_{1}, \ldots, T_{m-1}\right)\right)
$$

and $\operatorname{Gal}\left(f^{-}, k_{p}\left(T_{1}, \ldots, T_{e}\right)\right)$ has two or three orbits on $\mathcal{P}\left(G F(q)^{2 m}\right)$ of sizes $\left(q^{m}+1\right)\langle m-2\rangle, q^{m-1}\left(q^{m}+1\right)$ or $\left(q^{m}+1\right)\langle m-2\rangle, \quad q^{m-1}\left(q^{m}+1\right) / 2$, $q^{m-1}\left(q^{m}+1\right) / 2$ according as $p=2$ or $p \neq 2$.

Recall that a quasi-p group is a finite group which is generated by its $p$-Sylow subgroups. Since $\operatorname{Disc}_{Y} f_{e}^{-}=-1=\operatorname{Disc}_{Y} \phi_{e}^{-}$for $1 \leq e \leq m-1$, by the techniques of the proofs of Proposition 6 of [A01] and Lemma 34 of [A02] we get the following

Theorem (4.17). If $k_{p}$ is algebraically closed then, $\operatorname{Gal}\left(f_{e}^{-}, k_{p}\left(T_{1}, \ldots, T_{e}\right)\right)$ and $\operatorname{Gal}\left(\phi_{e}^{-}, k_{p}\left(T_{1}, \ldots, T_{e}\right)\right)$ for $1 \leq e \leq m-1$, are quasi-p groups. In particular, if $k_{p}$ is algebraically closed then, $\operatorname{Gal}\left(f^{-}, k_{p}\left(T_{1}, \ldots, T_{m-1}\right)\right)$ and $\operatorname{Gal}\left(\phi^{-}, k_{p}\left(T_{1}, \ldots, T_{m-1}\right)\right)$ are quasi-p groups. 


\section{Review of Linear Algebra}

Recall that we are assuming $m>1$. Let $\epsilon \in\{+,-\}$. Let $\epsilon^{\prime}=(1-\epsilon 1) / 2$ and note that then $\epsilon^{\prime}=0$ or 1 according as $\epsilon=+$ or - respectively.

Fix $\nu \in \operatorname{GF}(q)$ such that $T^{2}+T+\nu$ is irreducible in $\operatorname{GF}(q)[T]$. Consider the quadratic forms $\psi^{+}(x)=x_{1} x_{m+1}+\cdots+x_{m} x_{2 m}$ and $\psi^{-}(x)=x_{1} x_{m+1}+\cdots+$ $x_{m-1} x_{2 m-1}+x_{m}^{2}+x_{m} x_{2 m}+\nu x_{2 m}^{2}$. Define the orthogonal group $\mathrm{O}^{\epsilon}(2 m, q)$ as the group of all $e \in \mathrm{GL}(2 m, q)$ which leave the quadratic form $\psi^{\epsilon}$ unchanged, i.e., $\psi^{\epsilon}(x e)=\psi^{\epsilon}(x)$. Let the general orthogonal group $\mathrm{GO}^{\epsilon}(2 m, q)$ be defined as the group of all $e \in \operatorname{GL}(2 m, q)$ such that for some $\lambda(e) \in \operatorname{GF}(q)$ we have $\psi^{\epsilon}(\xi e)=\lambda(e) \psi^{\epsilon}(\xi)$ for all $\xi \in \mathrm{GF}(q)^{2 m}$. Let the semilinear orthogonal group $\Gamma \mathrm{O}^{\epsilon}(2 m, q)$ be defined as the group of all $(\tau, e) \in \Gamma \mathrm{L}(2 m, q)$, with $\tau \in \operatorname{Aut}(\operatorname{GF}(q))$ and $e \in \mathrm{GL}(2 m, q)$, such that for some $\lambda(\tau, e) \in \mathrm{GF}(q)$ we have $\psi^{\epsilon}\left(\xi^{\tau} e\right)=$ $\lambda(\tau, e) \psi^{\epsilon}(\xi)^{\tau}$ for all $\xi \in \operatorname{GF}(q)^{2 m}$. Define the special orthogonal group $\operatorname{SO}^{\epsilon}(2 m, q)=$ $\mathrm{SL}(2 m, q) \cap \mathrm{O}^{\epsilon}(2 m, q)$. Let $\mathrm{O}^{\prime} \epsilon(2 m, q)$ be the commutator subgroup of $\mathrm{O}^{\epsilon}(2 m, q)$. Let $\Omega^{\epsilon}(2 m, q)=\mathrm{O}^{\prime \epsilon}(2 m, q)$ if $(m, q, \epsilon) \neq(2,2,+)$, and let $\Omega^{+}(4,2)$ be the subgroup of $\mathrm{SO}^{+}(4,2)$ containing $\mathrm{O}^{\prime+}(4,2)$, as defined in Definition 4 on page 30 of [LiK], such that $\left[\mathrm{SO}^{+}(4,2): \Omega^{+}(4,2)\right]=2=\left[\Omega^{+}(4,2): \mathrm{O}^{\prime+}(4,2)\right]$. Thus we get the sequence $\mathrm{O}^{\prime}(2 m, q)<\Omega^{\epsilon}(2 m, q)<\mathrm{SO}^{\epsilon}(2 m, q)<\mathrm{O}^{\epsilon}(2 m, q)<\mathrm{GO}^{\epsilon}(2 m, q)<\Gamma \mathrm{O}^{\epsilon}(2 m, q)$ of orthogonal groups and by applying $\Theta_{2 m}$ to them we get the corresponding sequence $\mathrm{PO}^{\epsilon}(2 m, q)<\mathrm{P}^{\epsilon}(2 m, q)<\operatorname{PSO}^{\epsilon}(2 m, q)<\mathrm{PO}^{\epsilon}(2 m, q)<\operatorname{PGO}^{\epsilon}(2 m, q)<$ $\mathrm{P \Gamma O}^{\epsilon}(2 m, q)$ of projective orthogonal groups. ${ }^{3}$

Note that for any $H<\mathrm{GL}(2 m, q)$ we have

$$
\Omega^{\epsilon}(2 m, q)<H \Leftrightarrow \mathrm{P} \Omega^{\epsilon}(2 m, q)<\Theta_{2 m}(H)
$$

In case $(m, q, \epsilon) \neq(2,2,+)$, this follows exactly as in the proof of Lemma 2.3 of [A04] because then by Theorem 11.46 of [Tay] $\Omega^{\epsilon}(2 m, q)$ is generated by Siegel transformations. By the definition of a Siegel transformation (11.17 of [Tay]) we see that its order is $p$ or 1 , and the said proof is based on the fact that the group is generated by elements of $p$-power order, i.e., equivalently the fact that it is a quasi- $p$ group. So $(5.1)$ holds also for $(m, q, \epsilon)=(2,2,+)$ because by Proposition 2.9.1(iv) of $[\mathrm{LiK}] \Omega^{+}(4,2)$ is a quasi-2 group.

\footnotetext{
${ }^{3}$ Instead of taking the specific quadratic form $\psi^{\epsilon}$, in $[\mathrm{LiK}]$ these groups are defined for each quadratic form of "Witt defect $\epsilon$ ". Dickson [Dic] defines these groups for $p \neq 2$ by taking a different set of specific quadratic forms thus: if either $\epsilon=+$ and $q \equiv 1(\bmod 4)$ or $\epsilon=+$ and $q \equiv 3(\bmod 4)$ with $m$ even or $\epsilon=-$ and $q \equiv 3(\bmod 4)$ with $m$ odd then take the quadratic form to be $x_{1}^{2}+\cdots+x_{2 m}^{2}$; if either $\epsilon=+$ and $q \equiv 3(\bmod 4)$ with $m$ odd or $\epsilon=-$ and $q \equiv 3$ $(\bmod 4)$ with $m$ even then take the quadratic form to be $x_{1}^{2}+\cdots+x_{2 m-1}^{2}-x_{2 m}^{2}$; and finally if $\epsilon=-$ and $q \equiv 1(\bmod 4)$ then take the quadratic form to be $x_{1}^{2}+\cdots+x_{2 m-1}^{2}-\mu x_{2 m}^{2}$ with $\mu \in \mathrm{GF}(q) \backslash \mathrm{GF}(q)^{2}$. By the singular points of $\mathrm{P}^{\epsilon}(2 m, q)$ we mean the images in $\mathcal{P}\left(\mathrm{GF}(q)^{2 m}\right)$ of the nonzero $\xi \in \mathrm{GF}(q)^{2 m}$ at which the quadratic form vanishes. By Exercise 11.3 on page 174 of [Tay] we see that the cardinality of the singular points of $\mathrm{P} \Omega^{\epsilon}(2 m, q)$ is $\left(q^{m-1+\epsilon^{\prime}}+1\right)\left\langle m-1-\epsilon^{\prime}\right\rangle$, and hence the cardinality of the nonsingular points of $\mathrm{P} \Omega^{\epsilon}(2 m, q)$ is $q^{m-1}\left(q^{m}-1+2 \epsilon^{\prime}\right)$. By 11.24 and 11.27 on pages $150-151$ of [Tay] we see that $\mathrm{P} \Omega^{\epsilon}(2 m, q)$ acts transitively on its singular points, and by using Witt's Lemma (page 81 of [Asc]) we see that if $p=2$, then $\operatorname{P}^{\epsilon}(2 m, q)$ acts transitively on its nonsingular points, whereas if $p \neq 2$, then $\mathrm{P}^{\epsilon}(2 m, q)$ has two equal size orbits of nonsingular points. Finally, by the sixth line of Table 5.4.C on page 200 of [LiK] which starts with $\left.D_{l}^{ \pm}(q)\right)$, we see that if $m>3$ and $\Phi<\operatorname{PGL}(2 m, q)$ is isomorphic to $\operatorname{P} \Omega^{\epsilon}(2 m, q)$, then $\mathrm{P} \Omega^{\epsilon}(2 m, q)=\delta^{-1} \Phi \delta$ for some $\delta \in \operatorname{PGL}(2 m, q)$.
} 
By 2.1.B, 2.10.4(ii) and 2.10.6(i) of [LiK], for any $H<\mathrm{GL}(2 m, q)$ we have

$$
\Omega^{\epsilon}(2 m, q) \triangleleft H \Leftrightarrow \Omega^{\epsilon}(2 m, q)<H<\mathrm{GO}^{\epsilon}(2 m, q)
$$

and by 2.1.C of $[\mathrm{LiK}]$ we have

$$
\left[\operatorname{GO}^{\epsilon}(2 m, q): \Omega^{\epsilon}(2 m, q)\right] \begin{cases}\not \equiv 0(\bmod p) & \text { if } p>2 \\ =2 & \text { if } p=2 .\end{cases}
$$

Since $\Omega^{\epsilon}(2 m, q)$ is quasi- $p$, it is generated by the $p$-power elements of $\Omega^{\epsilon}(2 m, q) \mathrm{GF}(q)^{*}$, and hence these two subgroups have the same normalizer in $\operatorname{GL}(2 m, q)$. Also clearly $\operatorname{GF}(q)^{*}<\operatorname{GO}^{\epsilon}(2 m, q)$. Therefore by $(5.2)$, for any $G<$ $\operatorname{PGL}(2 m, q)$ we have

$$
\mathrm{P} \Omega^{\epsilon}(2 m, q) \triangleleft G \Leftrightarrow \mathrm{P} \Omega^{\epsilon}(2 m, q)<G<\operatorname{PGO}^{\epsilon}(2 m, q)
$$

and by (5.3) we get

$$
\left[\operatorname{PGO}^{\epsilon}(2 m, q): \mathrm{P}^{\epsilon}(2 m, q)\right] \begin{cases}\not \equiv 0(\bmod p) & \text { if } p>2 \\ =2 & \text { if } p=2 .\end{cases}
$$

Finally, since $\operatorname{GF}(q)^{*}<\operatorname{GO}^{\epsilon}(2 m, q)$, for any $H<\mathrm{GL}(2 m, q)$ we have

$$
H<\operatorname{GO}^{\epsilon}(2 m, q) \Leftrightarrow \Theta_{2 m}(H)<\operatorname{PGO}^{\epsilon}(2 m, q) .
$$

In view of Theorem IV of [CaK], by Corollary 1(iii) of Kantor [Kan] we get the following:

Theorem (5.7) [KANTOR]. Assume that $m>3$. Let $G$ be a transitive permutation group of Rank 3 with subdegrees $1, q\left(q^{m-2+\epsilon^{\prime}}+1\right)\left\langle m-2-\epsilon^{\prime}\right\rangle$ and $q^{2 m-2}$. Then the permuted set can be identified with the singular points of $P \Omega^{\epsilon}(2 m, q)$ so that $P \Omega^{\epsilon}(2 m, q)_{1} \triangleleft G<P \Gamma O^{\epsilon}(2 m, q)_{1}$ where $P \Omega^{\epsilon}(2 m, q)_{1}$ and $P \Gamma O^{\epsilon}(2 m, q)_{1}$ denote the permutation groups on the said singular points induced by $P \Omega^{\epsilon}(2 m, q)$ and $P \Gamma O^{\epsilon}(2 m, q)$ respectively.

For applying (5.7), we first prove the following

Lemma (5.8). Let $G<P G L(m, q)$ have orbits $\Delta_{1} \ldots, \Delta_{e}$ of sizes $d_{1}, \ldots, d_{e}$ on $\mathcal{P}\left(G F(q)^{m}\right)$, and note that then $\sum_{i=1}^{n} d_{i}=\langle m-1\rangle$. Assume that there is no positive integer $r<m$ together with a proper subset $\rho$ of $\{1, \ldots, e\}$ such that $\sum_{i \in \rho} d_{i}=$ $\langle r-1\rangle$. Also assume that there is no integral divisor $s>1$ of $m$ together with a disjoint partition $\sigma(1) \cup \cdots \cup \sigma(s)=\{1, \ldots, e\}$ of $\{1, \ldots, e\}$ into pairwise disjoint nonempty subsets $\sigma(1), \ldots, \sigma(s)$ such that for $1 \leq j \leq s$ we have $\sum_{i \in \sigma(j)} d_{i}=$ $\left(\begin{array}{l}s \\ j\end{array}\right)(q-1)^{j-1}\langle(m / s)-1\rangle^{j}$. Then $G$ acts faithfully on each of its orbits.

Namely, the first assumption implies that $\Theta_{m}^{-1}(G)$ does not map any proper subspace of $\mathrm{GF}(q)^{m}$ (of positive dimension $r<m$ ) onto itself. ${ }^{4}$ Therefore, regarding

\footnotetext{
${ }^{4}$ In view of this observation, by the last line of Table 5.4.A on page 199 of [LiK] which starts with $D_{l}^{ \pm}(q)$, we see that if $m=3$ and $\Phi<\operatorname{PGL}(2 m, q)$ is isomorphic to and has the same size orbits as $\mathrm{P} \Omega^{\epsilon}(2 m, q)$, then $\mathrm{P} \Omega^{\epsilon}(2 m, q)=\delta^{-1} \Phi \delta$ for some $\delta \in \operatorname{PGL}(2 m, q)$.
} 
$\mathcal{P}\left(\mathrm{GF}(q)^{m}\right)$ as the set of all 1-dimensional subspaces of $\operatorname{GF}(q)^{m}$, it follows that $\Delta_{1}$ spans $\operatorname{GF}(q)^{m}$. Let $\Psi=\left\{\gamma \in \Theta_{m}^{-1}(G): \gamma(M)=M\right.$ for all $\left.M \in \Delta_{1}\right\}$. Then $\Psi \triangleleft \Theta_{m}^{-1}(G)$. Recall that a maximal eigenspace of $\Psi$ is a maximal subspace $L$ of $\operatorname{GF}(q)^{m}$ such that for some homomorphism $\alpha_{L}: \Psi \rightarrow \operatorname{GF}(q)^{*}$ we have $\gamma(z)=$ $\alpha_{L}(\gamma) z$ for all $\gamma \in \Theta_{m}(\Psi)$ and $z \in L$. Since $\Delta_{1}$ spans $\operatorname{GF}(q)^{m}$, we get a direct sum decomposition $\operatorname{GF}(q)^{m}=L_{1}+\cdots+L_{s}$ where $L_{1}, \ldots, L_{s}$ are maximal eigenspaces of $\Psi$. Since $\Psi \triangleleft \Theta_{m}^{-1}(G)$, it follows that $\Theta_{m}^{-1}(G)$ acts transitively on this decomposition, and hence $\operatorname{dim} L_{i}=m / s$ for $1 \leq i \leq s$. For $1 \leq j \leq s$ let $\Lambda_{j}$ be the set of all $M \in \mathcal{P}\left(\mathrm{GF}(q)^{m}\right)$ such that, for every $0 \neq z \in M$, the cardinality of $\{1 \leq i \leq s$ : $\left.\operatorname{proj}_{i}(z) \neq 0\right\}$ is $j$ where $\operatorname{proj}_{i}: L_{1}+\cdots+L_{s} \rightarrow L_{i}$ is the natural projection. Then the cardinality of $\Lambda_{j}$ is $\left(\begin{array}{l}s \\ j\end{array}\right)(q-1)^{j-1}\langle(m / s)-1\rangle^{j}$. Since $\Theta_{m}^{-1}(G)$ acts transitively on the above decomposition, there is a disjoint partition $\sigma(1) \cup \cdots \cup \sigma(s)=\{1, \ldots, e\}$ of $\{1, \ldots, e\}$ such that for $1 \leq j \leq s$ we have $\Lambda_{j}=\cup_{i \in \sigma(j)} \Delta_{i}$. Therefore for $1 \leq j \leq s$ we have $\sum_{i \in \sigma(j)} d_{i}=\left(\begin{array}{l}s \\ j\end{array}\right)(q-1)^{j-1}\langle(m / s)-1\rangle^{j}$. Consequently by the second assumption we must have $s=1$. Therefore $\Psi=\operatorname{GF}(q)^{*}$ and hence $G$ acts faithfully on $\Delta_{1}$. Similarly $G$ acts faithfully on each of its orbits.

In view of (5.8) and the previous two footnotes, we get the following corollary of (5.7):

Corollary (5.9). Assume that $m>3$. Let $G<P G L(2 m, q)$ have 2 or 3 orbits on $\mathcal{P}\left(G F(q)^{2 m}\right)$ of sizes $\left(q^{m}+1\right)\langle m-2\rangle, q^{m-1}\left(q^{m}+1\right)$ or $\left(q^{m}+1\right)\langle m-2\rangle$, $q^{m-1}\left(q^{m}+1\right) / 2, q^{m-1}\left(q^{m}+1\right) / 2$ according as $p=2$ or $p \neq 2$. Assume that $G$ is Rank 3 with subdegrees $1, q\left(q^{m-2+\epsilon^{\prime}}+1\right)\left\langle m-2-\epsilon^{\prime}\right\rangle$ and $q^{2 m-2}$ on the orbit of size $\left(q^{m}+1\right)\langle m-2\rangle$. Then $P \Omega^{\epsilon}(2 m, q) \triangleleft \delta^{-1} G \delta$ for some $\delta \in P G L(2 m, q)$.

As in (5.7), let $\mathrm{P} \Omega^{\epsilon}(2 m, q)_{1}$ denote the permutation group induced by $\mathrm{P} \Omega^{\epsilon}(2 m, q)$ on its singular points (whose cardinality is $\left(q^{m}+1\right)\langle m-2\rangle$ ). In case of $p=$ 2 , let $\mathrm{P} \Omega^{\epsilon}(2 m, q)_{2}$ denote the permutation group induced by $\mathrm{P} \Omega^{\epsilon}(2 m, q)$ on its nonsingular points (whose cardinality is $q^{m-1}\left(q^{m}+1\right)$ ). In case of $p \neq 2$, the permutation groups induced by $\mathrm{P} \Omega^{\epsilon}(2 m, q)$ on its two nonsingular orbits (whose common cardinality is $\left.q^{m-1}\left(q^{m}+1\right) / 2\right)$ are easily seen to be equivalent and we denote them by $\mathrm{P} \Omega^{\epsilon}(2 m, q)_{2}$. Now by $(5.8)$ we see that

$$
\mathrm{P} \Omega^{\epsilon}(2 m, q)_{1} \approx \mathrm{P} \Omega^{\epsilon}(2 m, q) \approx \mathrm{P} \Omega^{\epsilon}(2 m, q)_{2}
$$

where $\approx$ denotes isomorphism as abstract groups.

\section{Galois Groups}

By (4.15), (4.16), (5.1), (5.6) and (5.9) we get the following

Theorem (6.1). If $m>3$ and $G F(q) \subset k_{p}$, then, for $2 \leq e \leq m-1$, in a natural manner, we have

$$
\Omega^{-}(2 m, q)<\operatorname{Gal}\left(\phi_{e}^{-}, k_{p}\left(T_{1}, \ldots, T_{e}\right)\right)<G O^{-}(2 m, q)
$$

and

$$
P \Omega^{-}(2 m, q)<\operatorname{Gal}\left(f_{e}^{-}, k_{p}\left(T_{1}, \ldots, T_{e}\right)\right)<P G O^{-}(2 m, q) .
$$

Hence in particular, if $m>3$ and $G F(q) \subset k_{p}$ then, in a natural manner we have

$$
\Omega^{-}(2 m, q)<\operatorname{Gal}\left(\phi^{-}, k_{p}\left(T_{1}, \ldots, T_{m-1}\right)\right)<G O^{-}(2 m, q)
$$


and

$$
P \Omega^{-}(2 m, q)<\operatorname{Gal}\left(f^{-}, k_{p}\left(T_{1}, \ldots, T_{m-1}\right)\right)<P G O^{-}(2 m, q) .
$$

By (3.0), (3.1), (3.4), (3.5), (4.17), (5.2), (5.3), (5.4), (5.5), (5.10) and (6.1) we get the following

Theorem (6.2). If $m>3 \leq p$ and $k_{p}$ is algebraically closed, then, for $2 \leq e \leq$ $m-1$, in a natural manner we have

$$
\operatorname{Gal}\left(\phi^{-}, k_{p}\left(T_{1}, \ldots, T_{m-1}\right)=\operatorname{Gal}\left(\phi_{e}^{-}, k_{p}\left(T_{1}, \ldots, T_{e}\right)=\Omega^{-}(2 m, q)\right.\right.
$$

and

$$
\operatorname{Gal}\left(f^{-}, k_{p}\left(T_{1}, \ldots, T_{m-1}\right)\right)=\operatorname{Gal}\left(f_{e}^{-}, k_{p}\left(T_{1}, \ldots, T_{e}\right)\right)=P \Omega^{-}(2 m, q)
$$

and

$$
\begin{aligned}
\operatorname{Gal}\left(\bar{f}, k_{p}\left(T_{1}, \ldots, T_{m-1}\right)\right) & =\operatorname{Gal}\left(\bar{f}_{e}, k_{p}\left(T_{1}, \ldots, T_{e}\right)\right) \\
& =P \Omega^{-}(2 m, q)_{1} \approx P \Omega^{-}(2 m, q)
\end{aligned}
$$

and

$$
\begin{aligned}
\operatorname{Gal}\left(f^{* *}, k_{p}\left(T_{1}, \ldots, T_{m-1}\right)\right) & =\operatorname{Gal}\left(f_{e}^{* *}, k_{p}\left(T_{1}, \ldots, T_{e}\right)\right) \\
& =P \Omega^{-}(2 m, q)_{2} \approx P \Omega^{-}(2 m, q)
\end{aligned}
$$

and

$$
\begin{aligned}
\operatorname{Gal}\left(f^{* * *}, k_{p}\left(T_{1}, \ldots, T_{m-1}\right)\right) & =\operatorname{Gal}\left(f_{e}^{* * *}, k_{p}\left(T_{1}, \ldots, T_{e}\right)\right) \\
& =P \Omega^{-}(2 m, q)_{2} \approx P \Omega^{-}(2 m, q) .
\end{aligned}
$$

Remark (6.3). We shall discuss the $m \leq 3$ or $p=2$ case elsewhere.

\section{REFERENCES}

[A01] S. S. Abhyankar, Coverings of algebraic curves, American Journal of Mathematics 79 (1957), 825-856. MR 20:872

[A02] S. S. Abhyankar, Tame coverings and fundamental groups of algebraic varieties, Part I, American Journal of Mathematics 81 (1959), 46-94. MR 21:3428

[A03] S. S. Abhyankar, Galois theory on the line in nonzero characteristic, Dedicated to "Feit-Serre-Email", Bulletin of the American Mathematical Society 27 (1992), 68-133. MR 94a:12004

[A04] S. S. Abhyankar, Nice equations for nice groups, Israel Journal of Mathematics 88 (1994), 1-24. MR 95:04

[A05] S. S. Abhyankar, More nice equations for nice groups, Proceedings of the American Mathematical Society (to appear).

[Asc] M. Aschbacher, Finite Group Theory, Cambridge University Press, 1986. MR 89b:20001

[BuS] F. Buekenhout and E. E. Shult, On the foundations of polar geometry, Geometriae Dedicata 3 (1974), 155-170. MR 50:3091

[CaK] P. J. Cameron and W. M. Kantor, 2-Transitive and antiflag transitive collineation groups of finite projective spaces, Journal of Algebra 60 (1979), 384-422. MR 81c:20032

[Dic] L. E. Dickson, Linear Groups, Teubner, 1901. 
[Kan] W. M. Kantor, Rank 3 characterizations of classical geometries, Journal of Algebra 36 (1975), 309-313. MR 52:8229

[LiK] M. W. Liebeck and P. Kleidman, The Subgroup Structure of the Finite Classical Groups, Cambridge University Press, 1990. MR 91g:20001

[Tay] D. E. Taylor, The Geometry of the Classical Groups, Heldermann Verlag, Berlin, 1992. MR 94d:20028

[Tit] J. Tits, Buildings of Spherical Type and Finite BN-Pairs, Springer Lecture Notes In Mathematics Number 386, 1974. MR 57:9866

Department of Mathematics, Purdue University, West Lafayette, Indiana 47907

E-mail address: ram@cs.purdue.edu 